\title{
CONJECTURES ABOUT $p$-ADIC GROUPS AND THEIR NONCOMMUTATIVE GEOMETRY
}

\author{
ANNE-MARIE AUBERT, PAUL BAUM, ROGER PLYMEN, AND MAARTEN SOLLEVELD
}

\begin{abstract}
Let $G$ be any reductive $p$-adic group. We discuss several conjectures, some of them new, that involve the representation theory and the geometry of $G$.

At the heart of these conjectures are statements about the geometric structure of Bernstein components for $G$, both at the level of the space of irreducible representations and at the level of the associated Hecke algebras. We relate this to two well-known conjectures: the local Langlands correspondence and the BaumConnes conjecture for $G$. In particular, we present a strategy to reduce the local Langlands correspondence for irreducible $G$-representations to the local Langlands correspondence for supercuspidal representations of Levi subgroups.
\end{abstract}

\section{Contents}

Introduction

1. The local Langlands correspondence 2

1.1. Tori

1.2. Quasi-split groups

1.3. Inner forms and inner twists

1.4. Enhanced L-parameters and relevance

1.5. A bijective version of the LLC

2. The smooth dual of a reductive $p$-adic group

2.1. Twisted extended quotients

2.2. The Bernstein decomposition

2.4. Hecke algebras for Bernstein blocks 20

2.5. Conjectural construction of the bijection 23

3. Reduction to the supercuspidal case 24

3.1. Towards a Galois analogue of the Bernstein theory 25

3.2. Langlands parameters and extended quotients 27

4. Topological K-theory 29

4.1. Equivariant K-theory 29

4.2. The Baum-Connes conjecture 30

4.3. Relations with the LLC 32

References

Date: January 3, 2016.

2010 Mathematics Subject Classification. 20G25, 22E50, 11S37, $19 \mathrm{~L} 47$. 


\section{INTRODUCTION}

This survey paper arose from talks that the first and fourth author gave at the conference "Around Langlands correspondences" in Orsay in June 2015. We discuss the representation theory of reductive $p$-adic groups from two different viewpoints: the Langlands program and noncommutative geometry. We do this with the aid of several conjectures.

In the first part we formulate a version of the (conjectural) local Langlands correspondence which is tailored for our purposes. In part 2 we explain what has become known as the ABPS conjecture. We phrase the most general version, for any reductive group over a local nonarchimedean field, not necessarily split. One of the foundations of this conjecture is the structure of the Hecke algebras associated to Bernstein components. Based on many known cases we describe in Conjecture 3 what these algebras should look like in general, up to Morita equivalence.

Part 3 focuses on the Galois side of the local Langlands correspondence (LLC). We conjecture that the space of enhanced L-parameters is in bijection with a certain union of extended quotients, analogous to the ABPS conjecture. This and Conjecture 3 have not appeared in print before. Together these conjectures provide a strategy to reduce the construction of a LLC for a reductive $p$-adic group to that for supercuspidal representations of its Levi subgroups.

The final part of the paper is purely noncommutative geometric. We discuss the ABPS conjecture for the topological K-theory of the reduced $C^{*}$-algebra of a reductive $p$-adic group. We show that it forms a bridge between the Baum-Connes conjecture and the LLC.

Acknowledgment. We thank the referee for several helpful comments.

\section{The local Langlands Correspondence}

We briefly discuss the history of the local Langlands correspondence (LLC). With a sequence of examplary groups we will reach more and more refined versions of the LLC. We will use these examples to explain exactly what kind of L-parameters we want to use, and we conjecture a bijective version of this correspondence.

The (local) Langlands program originated from two sources:

- (local) class field theory;

- representation theory of real reductive groups, in particular the work of Harish-Chandra on the discrete series.

Already in his 1973 preprint [Lan1] Langlands established his correspondence for real reductive groups: he managed to canonically associate an L-parameter to every (admissible, smooth) irreducible representation of such a group.

In this paper we focus entirely on the non-archimedean case, so let $F$ be either a $p$-adic field or a local function field. We fix a separable closure $F_{\text {sep }}$ and we let $\mathbf{W}_{F} \subset \operatorname{Gal}\left(F_{\text {sep }} / F\right)$ be the Weil group of $F$.

\subsection{Tori.}

Let $\mathbf{W}_{F}^{\text {ab }}:=\mathbf{W}_{F} / \overline{\left[\mathbf{W}_{F}, \mathbf{W}_{F}\right]}$ be the quotient of $\mathbf{W}_{F}$ by closure of its commutator subgroup. Recall that Artin reciprocity provides a natural isomorphism of topological groups

$$
\mathbf{a}_{F}: F^{\times} \rightarrow \mathbf{W}_{F}^{\mathrm{ab}} .
$$


Langlands had the beautiful idea to interpret this as a statement about $\mathrm{GL}_{1}(F)$ which admits generalization to other reductive groups. Namely, let $\operatorname{Irr}\left(F^{\times}\right)$be the collection of irreducible smooth complex representations of $F^{\times}$. Of course these are all characters, as $F^{\times}$is commutative. Composition with (1) gives a bijection

$$
\operatorname{Hom}\left(\mathbf{W}_{F}, \mathbb{C}^{\times}\right)=\operatorname{Hom}\left(\mathbf{W}_{F}^{\mathrm{ab}}, \mathbb{C}^{\times}\right) \stackrel{\sim}{\longrightarrow} \operatorname{Irr}\left(F^{\times}\right) .
$$

(Here and below "Hom" means smooth homomorphisms of topological groups.) More generally, suppose that $S=\mathcal{S}(F)$ is a $F$-split torus. Let $X^{*}(S)$ (resp. $X_{*}(S)$ ) be the lattice of algebraic characters $\mathcal{S} \rightarrow \mathrm{GL}_{1}$ (resp. algebraic cocharacters $\left.\mathrm{GL}_{1} \rightarrow \mathcal{S}\right)$. These two lattices are canonically dual to each other and

$$
S \cong X_{*}(\mathcal{S}) \otimes_{\mathbb{Z}} F^{\times} \cong\left(F^{\times}\right)^{\operatorname{dim} \mathcal{S}} \text {. }
$$

Let $S^{\vee}:=X^{*}(\mathcal{S}) \otimes_{\mathbb{Z}} \mathbb{C}^{\times}$be the complex dual torus of $S$, characterized by

$$
X^{*}\left(S^{\vee}\right)=X_{*}(\mathcal{S}), \quad X_{*}\left(S^{\vee}\right)=X^{*}(\mathcal{S}) .
$$

With Hom-tensor-duality (2) generalizes to

$$
\begin{aligned}
\operatorname{Irr}(S)= & \operatorname{Irr}\left(X_{*}(\mathcal{S}) \otimes_{\mathbb{Z}} F^{\times}\right)= \\
& \operatorname{Hom}\left(X_{*}(\mathcal{S}) \otimes_{\mathbb{Z}} F^{\times}, \mathbb{C}^{\times}\right) \cong \operatorname{Hom}\left(F^{\times}, X^{*}(\mathcal{S}) \otimes_{\mathbb{Z}} F^{\times}\right)= \\
& \operatorname{Hom}\left(F^{\times}, S^{\vee}\right) \stackrel{\sim}{\sim} \operatorname{Hom}\left(\mathbf{W}_{F}^{\mathrm{ab}}, S^{\vee}\right)=\operatorname{Hom}\left(\mathbf{W}_{F}, S^{\vee}\right) .
\end{aligned}
$$

Motivated by (3), a Langlands parameter for $S$ is defined to be a smooth group homomorphism $\mathbf{W}_{F} \rightarrow S^{\vee}$. The collection of such parameters is denoted $\Phi(S)$, so we can rephrase (3) as a natural bijection

$$
\operatorname{Irr}(S) \rightarrow \Phi(S)
$$

Already in 1968 Langlands generalized this to non-split tori. For example, let $E$ be a finite extension of $F$ contained in $F_{\text {sep }}$ and let $T=\operatorname{Res}_{E / F}\left(E^{\times}\right)$, that is, consider $E^{\times}$as $F$-group. From (4) we get a bijection $\operatorname{Irr}(T) \rightarrow \operatorname{Hom}\left(\mathbf{W}_{E}, \mathbb{C}^{\times}\right)$, and it is desirable to reformulate to right hand side in terms of $\mathbf{W}_{F}$. Recall that $\mathbf{W}_{E}$ is an open subgroup of $\mathbf{W}_{F}$ of index $[E: F]$. The complex dual group of $T=\mathcal{T}(F)$ is

$$
\mathcal{T}^{\vee}(\mathbb{C})=T^{\vee}=\operatorname{ind}_{\mathbf{W}_{E}}^{\mathbf{W}_{F}}\left(\mathbb{C}^{\times}\right)=\operatorname{Map}\left(\mathbf{W}_{F} / \mathbf{W}_{E}, \mathbb{C}^{\times}\right) .
$$

It is a complex torus of dimension $[E: F]=\operatorname{dim}_{F}(T)$ endowed with an action of $\mathbf{W}_{F}$ via left multiplication on $\mathbf{W}_{F} / \mathbf{W}_{E}$. According to Shapiro's lemma in continuous group cohomology

$$
\operatorname{Hom}\left(\mathbf{W}_{E}, \mathbb{C}^{\times}\right)=H_{c}^{1}\left(\mathbf{W}_{E}, \mathbb{C}^{\times}\right) \cong H_{c}^{1}\left(\mathbf{W}_{F}, \operatorname{ind}_{\mathbf{W}_{E}}^{\mathbf{W}_{F}}\left(\mathbb{C}^{\times}\right)=H_{c}^{1}\left(\mathbf{W}_{F}, T^{\vee}\right) .\right.
$$

Langlands [Lan2] showed that the composition of (3) and (5) is in fact true for every (non-split) torus $T=\mathcal{T}(F)$ : the group $T^{\vee}$ is always endowed with a canonical action of $\mathbf{W}_{F}$, and there is a natural bijection

$$
\operatorname{Irr}(T) \rightarrow H_{c}^{1}\left(\mathbf{W}_{F}, T^{\vee}\right) .
$$

In view of this $H_{c}^{1}\left(\mathbf{W}_{F}, T^{\vee}\right)$ is defined to be the space of Langlands parameters $\Phi(T)$, and (6) is known as the local Langlands correspondence for tori. More explicitly, $\Phi(T)$ consists of continuous group homomorphisms

$$
\phi: \mathbf{W}_{F} \rightarrow T^{\vee} \rtimes \mathbf{W}_{F} \quad \text { such that } \quad \phi(w) \in T^{\vee} w \forall w \in \mathbf{W}_{F} .
$$


Two such homomorphisms $\phi, \phi^{\prime}$ are considered equal in $H_{c}^{1}\left(\mathbf{W}, T^{\vee}\right)$ if they are conjugate by an element of $T^{\vee}$, that is, if there is a $t \in T^{\vee}$ such that

$$
\phi^{\prime}(w)=t \phi(w) t^{-1} \quad \forall w \in \mathbf{W}_{F} .
$$

\subsection{Quasi-split groups.}

The most fundamental case of the LLC is the group $\mathrm{GL}_{n}(F)$. According to Langlands' original scheme an L-parameter for this group should be an $n$-dimensional representation $\mathbf{W}_{F} \rightarrow \mathrm{GL}_{n}(\mathbb{C})$. However, the Bernstein-Zelevinsky classification [Zel] has shown that not all irreducible representations are obtained in this way. Comparing $l$-adic and complex representations of $\mathbf{W}_{F}$, Deligne [Del, §8] realized that $\mathbf{W}_{F}$ should be replaced by $\mathbf{W}_{F} \ltimes \mathbb{C}$ (now known as the Weil-Deligne group). Instead, we use the group $\mathbf{W}_{F} \times \mathrm{SL}_{2}(\mathbb{C})$ as a substitute of the Weil-Deligne group (which is possible, as explained in [Kna, §8] and [GrRe, Proposition 2.2]). Thus $\Phi\left(\mathrm{GL}_{n}(F)\right)$ is defined as the set of isomorphism classes of $n$-dimensional continuous representations

$$
\phi: \mathbf{W}_{F} \times \mathrm{SL}_{2}(\mathbb{C}) \rightarrow \mathrm{GL}_{n}(\mathbb{C})
$$

such that $\left.\phi\right|_{\mathrm{SL}_{2}(\mathbb{C})}$ is a homomorphism of algebraic groups. It was proven in [LRS] that for a local function field $F$ there is a canonical bijection

$$
\operatorname{rec}_{n, F}: \operatorname{Irr}\left(\mathrm{GL}_{n}(F)\right) \rightarrow \Phi\left(\mathrm{GL}_{n}(F)\right) .
$$

Later this result was also established when $F$ is a $p$-adic field [HaTa, Hen, Scho]. We note that all these proofs make use of global methods and of some very particular Shimura varieties, whose cohomology carries actions of groups related to $\operatorname{Gal}\left(F_{\text {sep }} / F\right)$ and $\mathrm{GL}_{n}(F)$. It has turned out to be very hard to find varieties which play an analogous role for other reductive groups.

What all the above groups have in common, is that the LLC is a canonical bijection from $\operatorname{Irr}(G)$ to $\Phi(G)$. This is false for almost any other group, for example, it already fails for $\mathrm{SL}_{2}(F)$. More refinements are needed to parametrize an L-packet (the set of representations that share the same L-parameter). We will introduce possible such refinements below.

But first we have to define precisely what we mean by a Langlands parameter for a general reductive $F$-group $G$. Let $G^{\vee}=\mathcal{G}^{\vee}(\mathbb{C})$ be the complex dual group, as in [Bor2, §2]. It is endowed with an action of $\operatorname{Gal}\left(F_{\text {sep }} / F\right)$, in a way which is canonical up to inner automorphisms of $G^{\vee}$. The group $G^{\vee} \rtimes \mathbf{W}_{F}$ is called (the Weil form of) the Langlands dual group ${ }^{L} G$. Its definition is canonical up to isomorphism.

From Artin reciprocity we see that Langlands parameters must involve smooth homomorphisms from the Weil group of $F$, and from the case of split tori we observe that the target must contain the complex dual group of $G$. In fact, the case of nonsplit tori forces us to take $G^{\vee} \rtimes \mathbf{W}_{F}$ as target and to consider $G^{\vee}$-conjugacy classes of homomorphisms. Finally, the case $\mathrm{GL}_{n}(F)$ shows that we should use $\mathbf{W}_{F} \times \mathrm{SL}_{2}(\mathbb{C})$ as the source of our homomorphisms. Through such considerations Borel [Bor2, $\S 8.2]$ arrived at the following notion.

Definition 1.1. A Langlands parameter (or L-parameter for short) $\phi$ for $G$ is smooth group homomorphism

$$
\phi: \mathbf{W}_{F} \times \mathrm{SL}_{2}(\mathbb{C}) \rightarrow G^{\vee} \rtimes \mathbf{W}_{F} \quad \text { such that: }
$$

- $\phi$ preserves the canonical projections to $\mathbf{W}_{F}$, that is, $\phi(w, x) \in G^{\vee} w$ for all $w \in \mathbf{W}_{F}$ and $x \in \mathrm{SL}_{2}(\mathbb{C})$; 
- $\phi(w)$ is semisimple for all $w \in \mathbf{W}_{F}$, that is, $\rho(\phi(w, x))$ is semisimple for every finite dimensional representation $\rho$ of $G^{\vee} \rtimes \mathbf{W}_{F}$;

- $\left.\phi\right|_{\mathrm{SL}_{2}(\mathbb{C})}: \mathrm{SL}_{2}(\mathbb{C}) \rightarrow G^{\vee}$ is a homomorphism of algebraic groups.

The group $G^{\vee}$ acts on the set $\tilde{\Phi}(G)$ of such $\phi$ 's by conjugation. The set of Langlands parameters for $G$ is defined as the set $\Phi(G)$ of $G^{\vee}$-orbits in $\tilde{\Phi}(G)$.

We note that $\Phi(G)$ is a subset of $H_{c}^{1}\left(\mathbf{W}_{F}, G^{\vee}\right)$. The conjectural local Langlands correspondence asserts that there exists a canonical, finite-to-one map

$$
\operatorname{Irr}(G) \rightarrow \Phi(G) .
$$

The inverse image of $\phi \in \Phi(G)$ is called the L-packet $\Pi_{\phi}(G)$. Given $\phi \in \tilde{\Phi}(G)$, let $Z_{G^{\vee}}(\phi)$ be the centralizer of $\phi\left(\mathbf{W}_{F} \times \mathrm{SL}_{2}(\mathbb{C})\right)$ in $G^{\vee}$. Notice that

$$
Z\left(G^{\vee}\right) \cap Z_{G^{\vee}}(\phi)=Z\left(G^{\vee}\right)^{\mathbf{W}_{F}}
$$

by the definition of $\phi$. The (geometric) R-group of $\phi$ is the component group

$$
\mathfrak{R}_{\phi}:=\pi_{0}\left(Z_{G^{\vee}}(\phi) / Z\left(G^{\vee}\right)^{\mathbf{W}_{F}}\right) .
$$

It is clear that, up to isomorphism, $\mathfrak{R}_{\phi}$ depends only on the image of $\phi$ in $\Phi(G)$.

Suppose now that $G$ is quasi-split over $F$. Then it is expected that $\Pi_{\phi}(G)$ is in bijection with $\operatorname{Irr}\left(\mathfrak{R}_{\phi}\right)$. This was first suggested in a special case in [Lus1, §1.5]. When $F$ is $p$-adic this was proven for quasi-split orthogonal and symplectic groups in [Art3], for corresponding quasi-split similitude groups in $[\mathrm{Xu}]$, and for quasi-split unitary groups in [Mok]. The main method in these works is twisted endoscopic transfer, they rely on the LLC for $\mathrm{GL}_{n}(F)$.

\subsection{Inner forms and inner twists.}

General connected reductive $F$-groups need not be quasi-split, but they are always forms of split $F$-groups. Let us recall the parametrization of forms by means of Galois cohomology. Two $F$-groups $G=\mathcal{G}(F)$ and $G_{2}=\mathcal{G}_{2}(F)$ are called forms of each other if $\mathcal{G}$ is isomorphic to $\mathcal{G}_{2}$ as algebraic groups, or equivalently if $\mathcal{G}\left(F_{\text {sep }}\right) \cong \mathcal{G}_{2}\left(F_{\text {sep }}\right)$ as

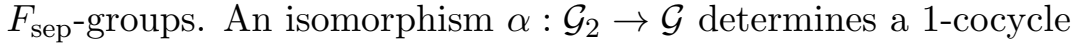

$$
\begin{aligned}
\gamma_{\alpha}: \operatorname{Gal}\left(F_{\mathrm{sep}} / F\right) & \rightarrow \operatorname{Aut}(\mathcal{G}) \\
\sigma & \mapsto \alpha \sigma \alpha^{-1} \sigma^{-1}
\end{aligned}
$$

From $\gamma_{\alpha}$ one can recover $G_{2}$ (up to isomorphism) as

$$
G_{2} \cong\left\{g \in \mathcal{G}\left(F_{\text {sep }}\right):\left(\gamma_{\alpha}(\sigma) \circ \sigma\right) g=g \quad \forall \sigma \in \operatorname{Gal}\left(F_{\text {sep }} / F\right)\right\} .
$$

Given another form $\beta: \mathcal{G}_{3} \rightarrow \mathcal{G}$, the groups $G_{2}$ and $G_{3}$ are $F$-isomorphic if and only if the 1-cocycles $\gamma_{\alpha}$ and $\gamma_{\beta}$ are cohomologous. That is, if there exists a $f \in \operatorname{Aut}(\mathcal{G})$ such that

$$
\gamma_{\alpha}(\sigma)=f^{-1} \gamma_{\beta}(\sigma) \sigma f \sigma^{-1} \quad \forall \sigma \in \operatorname{Gal}\left(F_{\text {sep }} / F\right) .
$$

In this way the isomorphism classes of forms of $G=\mathcal{G}(F)$ are in bijection with the Galois cohomology group $H^{1}(F, \operatorname{Aut}(\mathcal{G}))$. By definition $G_{2}$ is an inner form of $G$ if the cocycle $\gamma_{\alpha}$ takes values in the group of inner automorphisms $\operatorname{Inn}(\mathcal{G})$ (which is isomorphic to the adjoint group $\mathcal{G}_{\text {ad }}$ ). On the other hand, if the values of $\gamma_{\alpha}$ are not contained in $\operatorname{Inn}(\mathcal{G})$, then $G_{2}$ is called an outer form of $G$.

By $[$ Spr, $\S 16.4]$ that every connected reductive $F$-group is an inner form of a unique quasi-split $F$-group. It is believed that in the Langlands program it is advantageous to study all inner forms of a given group simultaneously. One reason is that the 
inner forms share the same Langlands dual group, because the action of $\mathbf{W}_{F}$ on $G^{\vee}$ is only uniquely defined up to inner automorphisms. Hence two inner forms have the same set of Langlands parameters. This also works the other way round: from the Langlands dual group ${ }^{L} G$ one can recover the inner form class of $G$.

Later we will see that it is even better to consider not inner forms, but rather inner twists of a fixed (quasi-split) group. An inner twist consists of a pair $\left(G_{2}, \alpha\right)$ as above, where $G_{2}=\mathcal{G}_{2}(F)$ and $\alpha: \mathcal{G}_{2} \stackrel{\sim}{\longrightarrow} \mathcal{G}$ are such that $\operatorname{im}\left(\gamma_{\alpha}\right) \subset \mathcal{G}_{\text {ad }}$. Two inner twists of $G$ are equivalent if (11) holds for some $f \in \operatorname{Inn}(\mathcal{G})$. The equivalence classes of inner twists of $G$ are parametrized by the Galois cohomology group $H^{1}\left(F, \mathcal{G}_{\text {ad }}\right)$.

It is quite possible that two inequivalent inner twists $\left(G_{2}, \alpha\right)$ and $\left(G_{3}, \beta\right)$ share the same group $G_{2} \cong G_{3}$. This happens precisely when $\gamma_{\alpha}$ and $\gamma_{\beta}$ are in the same orbit of $\operatorname{Aut}(\mathcal{G}) / \operatorname{Inn}(\mathcal{G})$ on $H^{1}\left(F, \mathcal{G}_{\text {ad }}\right)$.

Kottwitz has found an important alternative description of $H^{1}(F, \mathcal{G})$. Recall that the complex dual group $G^{\vee}=\mathcal{G}^{\vee}(\mathbb{C})$ is endowed with an action of $\operatorname{Gal}\left(F_{\text {sep }} / F\right)$.

Proposition 1.2. [Kot, Proposition 6.4]

There exists a natural isomorphism

$$
\kappa_{G}: H^{1}(F, \mathcal{G}) \stackrel{\sim}{\longrightarrow} \operatorname{Irr}\left(\pi_{0}\left(Z\left(G^{\vee}\right)^{\mathbf{W}_{F}}\right)\right)
$$

This is particularly useful in the following way. An inner twist of $G$ is the same thing as an inner twist of the unique quasi-split inner form $G^{*}=\mathcal{G}^{*}(F)$. Let $G_{\text {ad }}^{*}=\mathcal{G}_{\text {ad }}^{*}(F)$ be the adjoint group of $G^{*}$ and let $G_{\mathrm{sc}}^{\vee}=\left(G_{\mathrm{ad}}\right)^{\vee}$ be the simply connected cover of the derived group of $G^{\vee}$. Here the Kottwitz isomorphism becomes

$$
\kappa_{G_{\mathrm{ad}}^{*}}: H^{1}\left(F, \mathcal{G}_{\mathrm{ad}}^{*}\right) \stackrel{\sim}{\longrightarrow} \operatorname{Irr}\left(Z\left(G_{\mathrm{sc}}^{\vee}\right)^{\mathbf{W}_{F}}\right) .
$$

This provides a convenient way to parametrize inner twists of $G$.

Example. We work out the above when $\mathcal{G}=\mathrm{GL}_{n}$, relying on [Wei, §XI.4]. From (12) we see that

$$
H^{1}\left(F, \mathcal{G}_{\text {ad }}\right) \cong \operatorname{Irr}\left(Z\left(\mathrm{SL}_{n}(\mathbb{C})\right)\right)
$$

is cyclic of order $n$. Let $F_{(n)}$ be the unique unramified extension of $F$ of degree $n$, and let Frob $\in \mathbf{W}_{F}$ be an arithmetic Frobenius element. Thus

$$
\operatorname{Gal}\left(F_{\text {sep }} / F\right) / \operatorname{Gal}\left(F_{(n)} / F\right) \cong \operatorname{Gal}\left(F_{(n)} / F\right) \cong\langle\operatorname{Frob}\rangle /\left\langle\operatorname{Frob}^{n}\right\rangle \cong \mathbb{Z} / n \mathbb{Z} .
$$

Let $\varpi_{F}$ be a uniformizer and define $\gamma \in H^{1}\left(F, \mathrm{PGL}_{n}\right)$ by

$$
\gamma\left(\text { Frob }^{m}\right)=\theta_{n}^{m}, \quad \theta_{n}=\left(\begin{array}{cccc}
0 & & & \varpi_{F} \\
1 & 0 & & \\
& \ddots & \ddots & \\
& & 1 & 0
\end{array}\right) .
$$

Then $\gamma$ generates $H^{1}\left(F, \mathrm{PGL}_{n}\right)$. One can check that

$$
D_{\gamma}:=\left\{A \in M_{n}\left(F_{(n)}\right): \theta_{n} \operatorname{Frob}(A) \theta_{n}^{-1}=A\right\}
$$

is generated by $\theta_{n}$ and the matrices $\operatorname{diag}\left(a, \operatorname{Frob}(a), \ldots, \operatorname{Frob}^{n-1}(a)\right)$ with $a \in F_{(n)}$. It is a division algebra of dimension $n^{2}$ over its centre $F$, called the cyclic algebra $\left[F_{(n)} / F, \chi, \varpi_{F}\right]$ by Weil. The associated inner twist of $\mathrm{GL}_{n}(F)$ is

$$
\left(\mathrm{GL}_{1}\left(D_{\gamma}\right) \text {, inclusion } D_{\gamma}^{\times} \rightarrow \mathrm{GL}_{n}\left(F_{(n)}\right)\right)
$$


For $m<n, \gamma^{m} \in H^{1}\left(F, \mathrm{PGL}_{n}\right)$ is of order $d=n / \operatorname{gcd}(n, m)$. One obtains a division algebra $D_{\gamma^{m}}$ of dimension $d^{2}$ over $F$, contained in $M_{d}\left(F_{(d)}\right)$. The associated inner twist is

$$
\left(\operatorname{GL}_{\operatorname{gcd}(n, m)}\left(D_{\gamma^{m}}\right) \text {, inclusion in } \mathrm{GL}_{n}\left(F_{(d)}\right)\right) \text {. }
$$

In this way one finds that the inner twists of $\mathrm{GL}_{n}(F)$ are in bijection with the isomorphism classes of division algebras with centre $F$, whose dimension divides $n^{2}$.

Two inner forms $\mathrm{GL}_{m}(D)$ and $\mathrm{GL}_{m^{\prime}}\left(D^{\prime}\right)$ can be isomorphic even when $D$ is not isomorphic to $D^{\prime}$. For example let $D^{o p}$ be the opposite algebra of $D$ and denote the inverse transpose of a matrix $A$ by $A^{-T}$. Then

$$
\mathrm{GL}_{m}(D) \rightarrow \mathrm{GL}_{m}\left(D^{o p}\right): A \mapsto A^{-T}
$$

is a group isomorphism. The group $\operatorname{Aut}\left(\mathrm{GL}_{n}\right) / \operatorname{Inn}\left(\mathrm{GL}_{n}\right)$ has order two, the nontrivial element is represented by the inverse transpose map $-T$. The isomorphism (13) reflects the action of $\operatorname{Aut}\left(\mathrm{GL}_{n}\right) / \operatorname{Inn}\left(\mathrm{GL}_{n}\right)$ on $H^{1}\left(F, \mathrm{PGL}_{n}\right) \cong \mathbb{Z} / n \mathbb{Z}$ by $-T \cdot m=$ $-m$. Hence the isomorphism classes of inner forms of $\mathrm{GL}_{n}(F)$ are bijection with

$$
H^{1}\left(F, \operatorname{Inn}\left(\mathrm{GL}_{n}\right)\right) /\{1,-T\} \cong(\mathbb{Z} / n \mathbb{Z}) /\{ \pm 1\} .
$$

All the outer forms of $\mathrm{GL}_{n}(F)$ are unitary groups. If $\gamma: \operatorname{Gal}\left(F_{\text {sep }} / F\right) \rightarrow \operatorname{Aut}\left(\mathrm{GL}_{n}\right)$ is a non-inner 1-cocycle, then

$$
\operatorname{ker}\left(\operatorname{Gal}\left(F_{\mathrm{sep}} / F\right) \stackrel{\gamma}{\rightarrow} \operatorname{Aut}\left(\mathrm{GL}_{n}\right) / \operatorname{Inn}\left(\mathrm{GL}_{n}\right)\right)
$$

is an index two subgroup of $\operatorname{Gal}\left(F_{\text {sep }} / F\right)$. It defines a separable quadratic field extension $E / F$. One such cocycle is given by

$$
\gamma(\sigma)=\left\{\begin{array}{cl}
\text { id } & \sigma \in \operatorname{Gal}\left(F_{\text {sep }} / E\right), \\
\operatorname{Ad}\left(J_{n}\right) \circ-T & \sigma \in \operatorname{Gal}\left(F_{\text {sep }} / F\right) \backslash \operatorname{Gal}\left(F_{\text {sep }} / E\right)
\end{array} \quad J_{n}=\left(\begin{array}{cccc}
0 & \cdots & 0 & 1 \\
\vdots & . \cdot & 1 & 0 \\
0 & . \cdot & . \cdot & \vdots \\
1 & 0 & \cdots & 0
\end{array}\right)\right.
$$

The corresponding outer form $\mathrm{GL}_{n, \gamma}$ is

$$
\mathrm{U}_{n}(E / F):=\left\{A \in \operatorname{GL}_{n}(E):(\gamma(\sigma) \circ \sigma) A=A \quad \forall \sigma \in \operatorname{Gal}\left(F_{\mathrm{sep}} / F\right)\right\} .
$$

This is the unitary group associated with the Hermitian form on $E^{n}$ determined by $J_{n}$ and $F$. It is quasi-split, the upper triangular matrices in $\mathrm{U}_{n}(E / F)$ form a Borel subgroup. From (12) we see that

$$
\left|H^{1}\left(F, \mathrm{GL}_{n, \gamma}\right)\right|=\left|Z\left(\mathrm{SL}_{n}(\mathbb{C})\right)^{\mathbf{W}_{F}}\right|,
$$

where $\mathbf{W}_{F}$ acts on $\mathrm{SL}_{n}(\mathbb{C})$ via $\gamma$. Hence

$$
H^{1}\left(F, \mathrm{GL}_{n, \gamma}\right)=\left\{\begin{array}{cc}
\mathbb{Z} / 2 \mathbb{Z} & n \text { even } \\
1 & n \text { odd }
\end{array}\right.
$$

When $n$ is even, the unique other inner form of $U_{n}(E / F)$ is a unitary group associated to another $n$-dimensional Hermitian space over $E / F$. It can be constructed as above, but with a matrix $J_{n-2} \oplus\left(\begin{array}{ll}1 & 0 \\ 0 & a\end{array}\right)$ instead of $J_{n}$. Here $\left(\begin{array}{ll}1 & 0 \\ 0 & a\end{array}\right)$ represents a two-dimensional anisotropic Hermitian space. 


\subsection{Enhanced L-parameters and relevance.}

In spite of the successes for quasi-split classical groups, for more general groups, the R-group $\mathfrak{R}_{\phi}$ cannot always parametrize the L-packet $\Pi_{\phi}(G)$, this was already noticed in [Art2]. In fact, $\Pi_{\phi}(G)$ can very well be empty if $G$ is not quasi-split.

To overcome this problem, the notion of relevance of L-parameters was devised. It is derived from relevance of parabolic and Levi subgroups. (Below and later, we call a Levi factor of a parabolic subgroup of $G$ simply a Levi subgroup of $G$.) Let $\mathcal{T}$ be a maximal torus of $\mathcal{G}$ and let $\Delta$ be a basis of the root system $R(\mathcal{G}, \mathcal{T})$. Recall [Spr, Theorem 8.4.3] that the set of conjugacy classes of parabolic subgroups of $\mathcal{G}$ is in bijection with the power set of $\Delta$. The bijection

$$
R(\mathcal{G}, \mathcal{T}) \longleftrightarrow R^{\vee}(\mathcal{G}, \mathcal{T})=R\left(G^{\vee}, T^{\vee}\right)
$$

gives a basis $\Delta^{\vee}$, and provides a canonical bijection between the sets of conjugacy classes of parabolic subgroups of $\mathcal{G}$ and of $G^{\vee}$.

As in [Bor2, §3], we say that a parabolic subgroup $P^{\vee}$ of $G^{\vee}$ is $F$-relevant if the corresponding class of parabolic subgroups of $\mathcal{G}$ contains an element $\mathcal{P}$ which is defined over $F$. Similarly, we call a Levi subgroup $M^{\vee} \subset G^{\vee} F$-relevant if it is a Levi factor of a parabolic subgroup $P^{\vee} \subset G^{\vee}$ which is $F$-relevant.

We say that a parabolic subgroup $P^{\vee}$ of $G^{\vee}$ is quasi-stable under $\mathbf{W}_{F}$ if the projection $N_{G^{\vee} \rtimes \mathbf{W}_{F}}\left(P^{\vee}\right) \rightarrow \mathbf{W}_{F}$ is surjective. These are precisely the neutral components of what Borel [Bor2, §3] calls parabolic subgroups of $G^{\vee} \rtimes \mathbf{W}_{F}$.

Definition 1.3. Let $\phi \in \tilde{\Phi}(G)$ and let $P^{\vee}$ be a $\mathbf{W}_{F}$-quasi-stable parabolic subgroup of $G^{\vee}$ with a Levi factor $M^{\vee}$ such that

- the image of $\phi$ is contained in $N_{P \vee} \rtimes \mathbf{W}_{F}\left(M^{\vee}\right)$;

- $P^{\vee}$ is a minimal for this property.

Then $\phi$ is called relevant for $G$ if $P^{\vee}$ is F-relevant.

We remark that above one cannot substitute the first requirement by "the image of $\phi$ is contained in $P^{\vee} \rtimes \mathbf{W}_{F}$ ", that would give an unsatisfactory notion of relevance. It is expected that in general $\Pi_{\phi}(G)$ is nonempty if and only if $\phi$ is relevant for $G$.

Example. Let $G=D^{\times}$be the multiplicative group of a 4-dimensional noncommutative division algebra over $F$. It is the unique non-split inner form of $\mathrm{GL}_{2}(F)$. The only Levi subgroup of $D^{\times}$defined over $F$ is $D^{\times}$itself, and it corresponds to the Levi subgroup $\mathrm{GL}_{2}(\mathbb{C})$ on the complex side.

Consider $\phi_{1} \in \tilde{\Phi}\left(\mathrm{GL}_{2}(F)\right)=\tilde{\Phi}\left(D^{\times}\right)$which is just the embedding $\mathbf{W}_{F} \times \mathrm{SL}_{2}(\mathbb{C}) \rightarrow$ $\mathrm{GL}_{2}(\mathbb{C}) \times \mathbf{W}_{F}$. No proper parabolic subgroup of $\mathrm{GL}_{2}(\mathbb{C})$ contains $\phi_{1}\left(\mathrm{SL}_{2}(\mathbb{C})\right)=$ $\mathrm{SL}_{2}(\mathbb{C})$, so $\phi_{1}$ is relevant for both $D^{\times}$and $\mathrm{GL}_{2}(F)$. Indeed, $\Pi_{\phi}\left(\mathrm{GL}_{2}(F)\right)$ is the Steinberg representation of $\mathrm{GL}_{2}(F)$ and $\Pi_{\phi}\left(D^{\times}\right)$is the Steinberg representation of $D^{\times}$(which is just the trivial representation).

On the other hand, suppose that $\phi_{2} \in \tilde{\Phi}\left(\mathrm{GL}_{2}(\mathbb{C})\right)$ with

$$
\phi_{2}\left(\mathrm{SL}_{2}(\mathbb{C})\right)=1 \quad \text { and } \quad \phi_{2}\left(\mathbf{W}_{F}\right) \subset \operatorname{diag}\left(\mathrm{GL}_{2}(\mathbb{C})\right) \times \mathbf{W}_{F} .
$$

Then $M^{\vee}=\operatorname{diag}\left(\mathrm{GL}_{2}(\mathbb{C})\right)$ is the minimal Levi subgroup such that $M^{\vee} \times \mathbf{W}_{F}$ contains the image of $\phi_{2}$. Thus the standard Borel subgroup $P^{\vee}$ of $\mathrm{GL}_{2}(\mathbb{C})$ satisfies the conditions in Definition 1.3. But its conjugacy class does not correspond to any parabolic subgroup of $D^{\times}$, so $\phi_{2}$ is not relevant for $D^{\times}$. 
To parametrize L-packets, we must add some extra data to our Langlands parameters $\phi$. In view of the quasi-split case we need at least the irreducible representations of the geometric R-group $\mathfrak{R}_{\phi}$, but that is not enough. We will use enhancements that carry information about both the R-group of $\phi$ and the inner twists of $G$. We will follow Arthur's set-up in [Art2].

Recall that $G_{\mathrm{sc}}^{\vee}$ is the simply connected cover of both the derived group $G_{\mathrm{der}}^{\vee}$ and the adjoint group $G_{\text {ad }}^{\vee}$ of $G^{\vee}$. It acts on $\tilde{\Phi}(G)$ by conjugation, via the natural map $G_{\mathrm{sc}}^{\vee} \rightarrow G_{\mathrm{der}}^{\vee}$. For $\phi \in \tilde{\Phi}(G)$, let $Z_{G_{\mathrm{sc}}}(\phi)$ be the centralizer of $\phi\left(\mathbf{W}_{F} \times \mathrm{SL}_{2}(\mathbb{C})\right)$ in $G_{\mathrm{sc}}^{\vee}$. By $(8)$

$$
Z_{G^{\vee}}(\phi) / Z\left(G^{\vee}\right)^{\mathbf{W}_{F}} \cong Z_{G^{\vee}}(\phi) Z\left(G^{\vee}\right) / Z\left(G^{\vee}\right) .
$$

We can regard the right hand side as a subgroup of $G_{\text {ad }}^{\vee}$. Let $Z_{G_{\mathrm{sc}}^{\vee}}^{1}(\phi)$ be its inverse under the projection $G_{\mathrm{sc}}^{\vee} \rightarrow G_{\mathrm{ad}}^{\vee}$. Although $Z_{G_{\mathrm{sc}}^{\vee}}^{1}(\phi)$ contains $Z_{G_{\mathrm{sc}}^{\vee}}^{\vee}(\phi)$ as a normal subgroup of finite index, not all its elements fix $\phi$. More precisely

$$
Z_{G_{\mathrm{sc}}^{\vee}}^{1}(\phi)=\left\{g \in G_{\mathrm{sc}}^{\vee}: g \phi g^{-1}=\phi a_{g} \text { for some } a_{g} \in B^{1}\left(\mathbf{W}_{F}, Z\left(G^{\vee}\right)\right)\right\} .
$$

Here $B^{1}\left(\mathbf{W}_{F}, Z\left(G^{\vee}\right)\right)$ is the set of 1-coboundaries for group cohomology, that is, maps $\mathbf{W}_{F} \rightarrow Z\left(G^{\vee}\right)$ of the form $w \mapsto z w z^{-1} w^{-1}$ with $z \in Z\left(G^{\vee}\right)$.

The difference between $Z_{G_{\mathrm{sc}}^{\vee}}(\phi)$ and $Z_{G_{\mathrm{sc}}^{\vee}}^{1}(\phi)$ is caused by the identification (14), which as it were includes $Z\left(G^{\vee}\right)$ in $Z_{G^{\vee}}(\phi)$. We note that $Z_{G_{\mathrm{sc}}^{\vee}}^{1}(\phi)=Z_{G_{\mathrm{sc}}}(\phi)$ whenever $Z\left(G_{\mathrm{sc}}^{\vee}\right) \mathbf{W}_{F}=Z\left(G_{\mathrm{sc}}^{\vee}\right)$, in particular if $G$ is an inner twist of a split group. On the other hand, if $Z\left(G_{\mathrm{sc}}^{\vee}\right)^{\mathbf{W}_{F}} \neq Z\left(G_{\mathrm{sc}}^{\vee}\right)$, then it does not suffice to consider $Z_{G_{\mathrm{sc}}^{\vee}}(\phi)$, that would not necessarily account for all elements of $Z_{G^{\vee}}(\phi)$.

Definition 1.4. The $S$-group of $\phi$ is the component group $\mathcal{S}_{\phi}=\pi_{0}\left(Z_{G_{\mathrm{sc}}^{\vee}}^{1}(\phi)\right)$.

An enhancement of $\phi$ is an irreducible complex representation of $\mathcal{S}_{\phi}$.

The next lemma implies that every irreducible representation of $\mathfrak{R}_{\phi}$ lifts to one of $\mathcal{S}_{\phi}$.

Lemma 1.5. Write $\mathcal{Z}_{\phi}=Z\left(G_{\mathrm{sc}}^{\vee}\right) /\left(Z\left(G_{\mathrm{sc}}^{\vee}\right) \cap Z_{G_{\mathrm{sc}}^{\vee}}(\phi)^{\circ}\right)$. These groups fit in a natural central extension

$$
1 \rightarrow \mathcal{Z}_{\phi} \rightarrow \mathcal{S}_{\phi} \rightarrow \mathfrak{R}_{\phi} \rightarrow 1
$$

Proof. First we note that $Z\left(G_{\mathrm{sc}}^{\vee}\right)$ is contained in the centre of $Z_{G_{\mathrm{sc}}^{\vee}}^{1}(\phi)$. As $Z_{G_{\mathrm{sc}}^{\vee}}^{1}(\phi)^{\circ}=$ $Z_{G_{\mathrm{sc}}^{\vee}}(\phi)^{\circ}$, this means that $\mathcal{Z}_{\phi}$ is a central subgroup of $\mathcal{S}_{\phi}$.

The kernel of the natural map $Z_{G_{\mathrm{sc}}^{\vee}}^{1}(\phi) \rightarrow Z_{G^{\vee}}(\phi) Z\left(G^{\vee}\right) / Z\left(G^{\vee}\right)$ is $Z\left(G_{\mathrm{sc}}^{\vee}\right)$, so $\mathcal{Z}_{\phi}=\operatorname{ker}\left(\mathcal{S}_{\phi} \rightarrow \mathfrak{R}_{\phi}\right)$.

Consider any $g \in Z_{G^{\vee}}(\phi)$. Pick $g_{1} \in G_{\text {der }}^{\vee}$ and $g_{2} \in Z\left(G^{\vee}\right)^{\circ}$ so that $g_{2} g_{1}=g$. For any $(w, x) \in \mathbf{W}_{F} \times \mathrm{SL}_{2}(\mathbb{C})$ we have $\phi(w, x) \in G^{\vee} w$ and

$$
\begin{array}{r}
g_{1}^{-1} g_{2}^{-1}=g^{-1}=\phi(w, x) g^{-1} \phi(w, x)=\phi(w, x) g_{1}^{-1} \phi(w, x)^{-1} \phi(w, x) g_{2}^{-1} \phi(w, x)^{-1} \\
=\phi(w, x) g_{1}^{-1} \phi(w, x)^{-1} w g_{2}^{-1} w^{-1} .
\end{array}
$$

Hence $g_{1} \phi(w, x) g_{1}^{-1} \phi(w, x)^{-1}=g_{2}^{-1} w g_{2} w^{-1} \in Z\left(G^{\vee}\right)^{\circ} \cap G_{\mathrm{der}}^{\vee}$. In other words,

$$
g_{1} \phi(w, x) g_{1}^{-1}=\phi(w, x) a(w) \text { where } a(w)=g_{2}^{-1} w g_{2} w^{-1} \text {. }
$$

Let $g_{3} \in G_{\mathrm{sc}}^{\vee}$ be a lift of $g_{1} \in G_{\mathrm{der}}^{\vee}$. Then also $g_{3} \phi g_{3}^{-1}=\phi a$, showing that $g_{3} \in$ $Z_{G_{\mathrm{sc}}^{\vee}}^{1}(\phi)$. The image of $g_{3}$ in $Z_{G^{\vee}}(\phi) Z\left(G^{\vee}\right) / Z\left(G^{\vee}\right)$ is $g_{1} Z\left(G^{\vee}\right)=g_{2} g_{1} Z\left(G^{\vee}\right)=$ $g Z\left(G^{\vee}\right)$. Thus $\mathcal{S}_{\phi} \rightarrow \mathfrak{R}_{\phi}$ is surjective. 
Let us write $\mathcal{Z}_{\phi}^{\mathbf{W}_{F}}=Z\left(G_{\mathrm{sc}}^{\vee}\right)^{\mathbf{W}_{F}} /\left(Z\left(G_{\mathrm{sc}}^{\vee}\right)^{\mathbf{W}_{F}} \cap Z_{G_{\mathrm{sc}}}(\phi)^{\circ}\right)$. According to [Art2, §4]

$$
Z\left(G_{\mathrm{sc}}^{\vee}\right) \cap Z_{G_{\mathrm{sc}}}(\phi)^{\circ} \subset Z\left(G_{\mathrm{sc}}^{\vee}\right)^{\mathbf{W}_{F}} .
$$

Hence $\mathcal{Z}_{\phi}^{\mathbf{W}_{F}}$ can be regarded as a subgroup of $\mathcal{Z}_{\phi}$ and

$$
\mathcal{Z}_{\phi} / \mathcal{Z}_{\phi}^{\mathbf{W}_{F}} \cong Z\left(G_{\mathrm{sc}}^{\vee}\right) / Z\left(G_{\mathrm{sc}}^{\vee}\right)^{\mathbf{W}_{F}}
$$

By Schur's lemma every enhanced Langlands parameter $(\phi, \rho)$ restricts to a character $\left.\rho\right|_{\mathcal{Z}_{\phi} \mathbf{w}_{F}}$ of $\mathcal{Z}_{\phi}^{\mathbf{W}_{F}}$. This can be inflated to a character $\zeta_{\rho}$ of $Z\left(G_{\mathrm{sc}}^{\vee}\right) \mathbf{W}_{F}$. With the Kottwitz isomorphism (12) we get an element $\kappa_{G_{\text {ad }}^{*}}^{-1}\left(\zeta_{\rho}\right) \in H^{1}\left(F, \mathcal{G}_{\text {ad }}^{*}\right)$. In this way $(\phi, \rho)$ determines a unique inner twist of $G$. This can be regarded as an alternative way to specify for which inner twists of $G$ an enhanced Langlands parameter is relevant. Fortunately, it turns out that it agrees with the earlier definition of relevance of Langlands parameters.

Proposition 1.6. Let $\zeta \in \operatorname{Irr}\left(Z\left(G_{\mathrm{sc}}^{\vee}\right)^{\mathbf{W}_{F}}\right)$ and let $G_{\gamma}$ be the inner twist of $G$ associated to $\gamma=\kappa_{G_{\mathrm{ad}}^{*}}^{-1}(\zeta)$ via $(12)$. For $\phi \in \tilde{\Phi}(G)$ the following are equivalent:

(1) $\phi$ is relevant for $G_{\gamma}$;

(2) $Z\left(G_{\mathrm{sc}}^{\vee}\right)^{\mathbf{W}_{F}} \cap Z_{G_{\mathrm{sc}}}(\phi)^{\circ} \subset \operatorname{ker} \zeta$;

(3) there exists a $\rho \in \operatorname{Irr}\left(\mathcal{S}_{\phi}\right)$ such that $\zeta$ is the lift of $\left.\rho\right|_{\mathcal{Z}_{\phi}} ^{\mathbf{w}_{F}}$ to $Z\left(G_{\mathrm{sc}}^{\vee}\right)^{\mathbf{W}_{F}}$.

Proof. (1) $\Longleftrightarrow(2)$ See [HiSa, Lemma 9.1] and [Art1, Corollary 2.3]. We note that what Hiraga and Saito call $S_{\phi}^{\circ}$ equals $Z_{G_{\mathrm{sc}}^{\vee}}(\phi)^{\circ}$.

(2) $\Longrightarrow(3)$ Obvious.

$(2) \Longleftarrow(3)$ The assumption says that $\zeta$ can be regarded as a character of $\mathcal{Z}_{\phi}^{\mathbf{W}_{F}}$. The induced $\mathcal{S}_{\phi}$-representation ind $\mathcal{\mathcal { Z }}_{\mathcal{Z}_{\phi}}^{\mathbf{W}_{F}}(\zeta)$ has finite dimension and $Z\left(G_{\mathrm{sc}}^{\vee}\right)^{\mathbf{W}_{F}}$ acts on it as $\zeta$. Let $\rho$ be any irreducible constituent of $\operatorname{ind}_{\mathcal{Z}_{\phi}^{\mathbf{w}_{F}}}^{\mathcal{S}_{\phi}}(\zeta)$.

Supported by the above result, we extend the definition of relevance to inner twists and enhanced L-parameters.

Definition 1.7. Let $(G, \alpha)$ be an inner twist of a quasi-split F-group $G^{*}$. Let $\phi \in \tilde{\Phi}\left(G^{*}\right)=\tilde{\Phi}(G)$ and let $\rho \in \operatorname{Irr}\left(\mathcal{S}_{\phi}\right)$. We call $\rho$ relevant for $(G, \alpha)$ if

$$
\kappa_{G_{\mathrm{ad}}^{*}}^{-1}\left(\zeta_{\rho}\right)=\gamma_{\alpha}
$$

where $\zeta_{\rho}=\left.\rho\right|_{\mathcal{Z}_{\phi} \mathbf{w}_{F}}$ and $\gamma_{\alpha} \in H^{1}\left(F, \mathcal{G}_{\mathrm{ad}}^{*}\right)$ is defined in (10).

We denote the space of such relevant pairs $(\phi, \rho)$ by $\Phi_{e}(G)$. The group $G_{\mathrm{sc}}^{\vee}$ acts on $\tilde{\Phi}_{e}(G)$ by

$$
g \cdot(\phi, \rho)=\left(g \phi g^{-1}, g \cdot \rho\right), \text { where }(g \cdot \rho)\left(g h g^{-1}\right)=\rho(h) \text { for } h \in Z_{G_{\mathrm{sc}}^{\vee}}(\phi) .
$$

$A G_{\mathrm{sc}}^{\vee}$-orbit in $\tilde{\Phi}_{e}(G)$ is called an enhanced L-parameter for $G$, and the set of those is denoted $\Phi_{e}(G)$. 


\subsection{A bijective version of the LLC.}

We are ready to formulate our version of the conjectural local Langlands correspondence. It is inspired by many sources, in particular [Bor2, §10], [Vog, §4], [Art2, $\S 3]$ and [Hai, §5.2].

In some cases $\mathcal{S}_{\phi}$ is too large, because we have included the entire group $Z\left(G_{\mathrm{sc}}^{\vee}\right)$. To compensate for this it is handy to restrict our enhancements of L-parameters to a subset of $\operatorname{Irr}\left(\mathcal{S}_{\phi}\right)$. By Lemma 1.5 and Schur's lemma, the enhancement $\rho$ restricts to a character of $\mathcal{Z}_{\phi}^{\mathbf{W}_{F}}$, which then inflates to a character $\zeta_{\rho}$ of $Z\left(G_{\mathrm{sc}}^{\vee}\right)^{\mathbf{W}_{F}}$. If $\rho$ is relevant for $G$, then $\zeta_{\rho}=\kappa_{G_{\text {ad }}^{*}}\left(\gamma_{\alpha}\right) \in \operatorname{Irr}\left(Z\left(G_{\mathrm{sc}}^{\vee}\right)^{\mathbf{W}_{F}}\right)$. It can be extended in precisely $\left[Z\left(G_{\mathrm{sc}}^{\vee}\right): Z\left(G_{\mathrm{sc}}^{\vee}\right)^{\mathbf{W}_{F}}\right]$ ways to a character of $Z\left(G_{\mathrm{sc}}^{\vee}\right)$. We choose such an extension and we denote it by $\zeta_{G}$. By Proposition 1.6 every $\phi \in \tilde{\Phi}(G)$ can be enhanced with a $\rho \in \operatorname{Irr}\left(\mathcal{S}_{\phi}\right)$ such that $\left.\rho\right|_{\mathcal{Z}_{\phi}}$ inflates to $\zeta_{G}$.

We denote the set of equivalence classes of $\operatorname{such}(\phi, \rho) \in \tilde{\Phi}_{e}(G)$ by $\Phi_{e, \zeta_{G}}(G)$. Of course we pick $\zeta_{G}=\operatorname{triv}$ when $G$ is quasi-split. In that case Lemma 1.5 shows that $\Phi_{e, \text { triv }}(G)$ agrees with the set of enhanced L-parameters for $G$ discussed in Paragraph 1.2 .

Conjecture 1. Let $(G, \alpha)$ be an inner twist of a quasi-split F-group. There exists a surjection

$$
\Phi_{e}(G) \longrightarrow \operatorname{Irr}(G):(\phi, \rho) \mapsto \pi_{\phi, \rho},
$$

which becomes bijective when restricted to $\Phi_{e, \zeta_{G}}(G)$. We write its inverse as

$$
\operatorname{Irr}(G) \longrightarrow \Phi_{e, \zeta_{G}}(G): \pi \mapsto\left(\phi_{\pi}, \rho_{\pi}\right) .
$$

Then the composed map $\operatorname{Irr}(G) \rightarrow \Phi(G): \pi \mapsto \phi_{\pi}$ is canonical. These maps satisfy the properties (1) - (7) listed below.

We remark that the above bijection becomes more elegant if one considers the union over inner twists, then it says that there exists a surjection

$\left\{(\phi, \rho): \phi \in \Phi\left(G^{*}\right), \rho \in \operatorname{Irr}\left(\mathcal{S}_{\phi}\right)\right\} \rightarrow\left\{(G, \alpha, \pi):(G, \alpha)\right.$ inner twist of $\left.G^{*}, \pi \in \operatorname{Irr}(G)\right\}$ whose fibers have exactly $\left[Z\left(G_{\mathrm{sc}}^{\vee}\right): Z\left(G_{\mathrm{sc}}^{\vee}\right)^{\mathbf{W}_{F}}\right]$ elements.

Before we write down the additional properties, we recall two notions for L-parameters. Let $\phi \in \tilde{\Phi}(G)$. We say that $\phi$ is discrete (or elliptic) if there is no proper $\mathbf{W}_{F}$-stable Levi subgroup $M^{\vee} \subset G^{\vee}$ such that $\phi\left(\mathbf{W}_{F} \times \mathrm{SL}_{2}(\mathbb{C})\right) \subset M^{\vee} \rtimes \mathbf{W}_{F}$. We call $\phi$ bounded if $\phi^{\prime}\left(\mathbf{W}_{F}\right) \subset G^{\vee}$ is bounded, where $\phi(w)=\left(\phi^{\prime}(w), w\right)$. (This is equivalent to $\phi^{\prime}$ (Frob) being a compact element of $G^{\vee}$.)

\section{Desiderata for the local Langlands correspondence (Borel).}

(1) The central character of $\pi$ equals the character of $Z(G)$ constructed from $\phi_{\pi}$ in [Bor2, $\S 10.1]$.

(2) Let $z \in H_{c}^{1}\left(\mathbf{W}_{F}, Z\left(G^{\vee}\right)\right)$ be a class in continuous group cohomology, and let $\chi_{z}: G \rightarrow \mathbb{C}^{\times}$be the character associated to it in [Bor2, §10.2]. Thus $z \phi_{\pi} \in \tilde{\Phi}(G)$ and $\mathcal{S}_{z \phi_{\pi}}=\mathcal{S}_{\phi_{\pi}}$. Then the LLC should satisfy $\left(z \phi_{\pi}, \rho_{\pi}\right)=$ $\left(\phi_{\chi_{z} \pi}, \rho_{\chi_{z} \pi}\right)$.

(3) $\pi$ is essentially square-integrable if and only if $\phi_{\pi}$ is discrete.

(4) $\pi$ is tempered if and only if $\phi_{\pi}$ is bounded.

(5) Let $P$ be a parabolic subgroup of $G$ with Levi factor $M$. Suppose that $g \in N_{G}(M)$ and $\check{g} \in N_{G^{\vee}}\left(M^{\vee}\right)$ are such that $\operatorname{Ad}(g): M \rightarrow M$ and $\operatorname{Ad}(\check{g})$ : 
$M^{\vee} \rightarrow M^{\vee}$ form a corresponding pair of homomorphisms, in the sense of [Bor2, §2]. Then

$$
\left(\phi_{g \cdot \pi}, \rho_{g \cdot \pi}\right)=\left(\operatorname{Ad}(\check{g}) \phi_{\pi}, \check{g} \cdot \rho_{\pi}\right) \quad \text { for all } \quad \pi \in \operatorname{Irr}(M) .
$$

(6) Suppose that $\left(\phi^{M}, \rho^{M}\right) \in \Phi_{e}(M)$ is bounded. Then

$$
\left\{\pi_{\phi, \rho}: \phi=\phi^{M} \text { composed with }{ }^{L} M \rightarrow{ }^{L} G,\left.\rho\right|_{\mathcal{S}_{\phi}^{M}} \text { contains } \rho^{M}\right\}
$$

equals the set of irreducible constituents of the parabolically induced representation $I_{P}^{G}\left(\pi_{\phi^{M}, \rho^{M}}\right)$.

(7) If $\phi^{M}$ is discrete but not necessarily bounded, then (17) is the set of Langlands constituents of $I_{P}^{G}\left(\pi_{\phi^{M}, \rho^{M}}\right)$, as in [ABPS1, p. 30].

We note that in order to establish Conjecture 1 for (a collection of) groups, it suffices to prove it for tempered representations and bounded enhanced L-parameters. This follows from comparing the geometry of the spaces $\operatorname{Irr}(G)$ and $\Phi_{e}(G)$ [ABPS2], or from the Langlands classification for $\operatorname{Irr}(G)$ [Ren, $\S \mathrm{VII} .4]$ and its counterpart for L-parameters [SiZi].

Of course one can hope for many more properties, like compatibility with Lfunctions, adjoint $\gamma$-factors [HII] and functoriality. For our survey (1)-(7) are sufficient. This bijective version of the LLC, including the listed properties, is known in the following cases:

- General linear groups over division algebras, or more precisely inner twists of $\mathrm{GL}_{n}(F)$. It is a consequence of the LLC for $\mathrm{GL}_{n}(F)$ and the JacquetLanglands correspondence [DKV, Bad], see [ABPS3, Theorem 2.2].

- Inner twists of $\mathrm{SL}_{n}(F)$, see [HiSa, $\left.\S 12\right]$ and [ABPS3, Theorem 3.3].

- Orthogonal and symplectic groups [Art3] and similitude groups [Xu].

- Unitary groups [Mok, KMSW].

- Principal series representations of split groups [ABPS6, §16].

- Unipotent representations of adjoint groups [Lus6].

- Epipelagic representations of tamely ramified groups [Kal].

The last three items concern particular classes of representations of certain groups. All the groups for which the complete LLC is currently known are linked to $\mathrm{GL}_{n}(F)$, and the proofs for these groups use the LLC for general linear groups in an essential way. It appears to be a big challenge to find an approach to the LLC which does not rely on the case of $\mathrm{GL}_{n}(F)$, and can be applied to more general reductive groups.

\section{The SMooth DUAL of A Reductive $p$-ADiC GRouP}

Let $G$ be a connected reductive group over a local non-archimedean field, and let $\operatorname{Irr}(G)$ be the set of irreducible (smooth, complex) $G$-representations. In this section we discuss the geometric structure of $\operatorname{Irr}(G)$. It is topologized via the Jacobson topology for the Hecke algebra of $G$, and in this way it is automatically rather close to an algebraic variety. We propose a generalization of our earlier conjectures [ABP, ABPS2], which make the structure of $\operatorname{Irr}(G)$ much more precise. To formulate these conjectures, we need extended quotients and the Bernstein decomposition.

\subsection{Twisted extended quotients.}

Let $\Gamma$ be a group acting on a topological space $X$. In [ABPS6, $\S 2$ ] we studied various extended quotients of $X$ by $\Gamma$. In this paper we need the most general version, the twisted extended quotients. 
Let $\downarrow$ be a given function which assigns to each $x \in X$ a 2-cocycle

$$
\natural_{x}: \Gamma_{x} \times \Gamma_{x} \rightarrow \mathbb{C}^{\times} \text {, where } \Gamma_{x}=\{\gamma \in \Gamma: \gamma x=x\} .
$$

Recall that the twisted group algebra $\mathbb{C}\left[\Gamma_{x}, \bigsqcup_{x}\right]$ has a basis $\left\{N_{\gamma}: \gamma \in \Gamma_{x}\right\}$ and multiplication rules

$$
N_{\gamma} N_{\gamma^{\prime}}=\natural_{x}\left(\gamma, \gamma^{\prime}\right) N_{\gamma \gamma^{\prime}} \quad \gamma, \gamma^{\prime} \in \Gamma_{x} .
$$

It is assumed that $\natural_{\gamma x}$ and $\gamma_{*} \natural_{x}$ define the same class in $H^{2}\left(\Gamma_{\gamma x}, \mathbb{C}^{\times}\right)$, where $\gamma_{*}$ : $\Gamma_{x} \rightarrow \Gamma_{\gamma x}$ sends $\alpha$ to $\gamma \alpha \gamma^{-1}$. We define

$$
\widetilde{X}_{\natural}:=\left\{(x, \rho): x \in X, \rho \in \operatorname{Irr} \mathbb{C}\left[\Gamma_{x}, \natural_{x}\right]\right\} .
$$

and we topologize it by decreeing that a subset of $\widetilde{X}_{\natural}$ is open if and only if its projection to the first coordinate is open in $X$.

We require, for every $(\gamma, x) \in \Gamma \times X$, a definite algebra isomorphism

$$
\phi_{\gamma, x}: \mathbb{C}\left[\Gamma_{x}, \natural_{x}\right] \rightarrow \mathbb{C}\left[\Gamma_{\gamma x}, \natural_{\gamma x}\right]
$$

such that:

- if $\gamma x=x$, then $\phi_{\gamma, x}$ is conjugation by an element of $\mathbb{C}\left[\Gamma_{x}, \natural_{x}\right]^{\times}$;

- $\phi_{\gamma^{\prime}, \gamma x} \circ \phi_{\gamma, x}=\phi_{\gamma^{\prime} \gamma, x}$ for all $\gamma^{\prime}, \gamma \in \Gamma, x \in X$.

Then we can define a $\Gamma$-action on $\tilde{X}_{\natural}$ by

$$
\gamma \cdot(x, \rho)=\left(\gamma x, \rho \circ \phi_{\gamma, x}^{-1}\right) .
$$

We form the twisted extended quotient

$$
(X / / \Gamma)_{\natural}:=\tilde{X}_{\natural} / \Gamma .
$$

Notice that the data used to construct this are very similar to a 2-cocycle $z$ of $\Gamma$ with values in the continuous functions $X \rightarrow \mathbb{C}^{\times}$. By formulating it in the above way, we remove the need to define $z\left(\gamma, \gamma^{\prime}\right)$ at points of $X$ that are not fixed by $\gamma$.

Furthermore we note that $(X / / \Gamma)_{\natural}$ reduces to the extended quotient of the second kind $(X / / \Gamma)_{2}$ from [ABPS6, §2] if $\natural_{x}$ is trivial for all $x \in X$ and $\phi_{\gamma, x}$ is conjugation by $\gamma$.

The extended quotient of the second kind is an extension of the ordinary quotient in the sense that it keeps track of the duals of the isotropy groups. Namely, in $(X / / \Gamma)_{2}$ every point $x \in X / \Gamma$ has been replaced by the set $\operatorname{Irr}\left(\Gamma_{x}\right)$.

In the context of representation theory, the twisted extended quotient comes into play when reducibility at a point is less than expected. To be precise, the number of inequivalent irreducible representations at a point is fewer than expected.

Example. Let $\Gamma=\{ \pm 1\}^{2}$, acting on the square $X=[-1,1]^{2}$ by sign changes of the coordinates. In the extended quotient $(X / / \Gamma)_{2}$ we have two points laying over $(x, 0)$ and over $(0, y)$, since $\Gamma_{(x, 0)} \cong \Gamma_{(0, y)} \cong \mathbb{Z} / 2 \mathbb{Z}$. The fiber over $(0,0)$ even has four points, because

$$
\mathbb{C}\left[\Gamma_{(0,0)}\right]=\mathbb{C}[\Gamma] \cong \mathbb{C}^{4}
$$

We define a nontrivial 2-cocycle of $\Gamma$ as follows. Define a projective $\Gamma$-representation $\lambda$ on $\mathbb{C}^{2}$ by

$$
\lambda(1,1)=\left(\begin{array}{ll}
1 & 0 \\
0 & 1
\end{array}\right), \lambda(1,-1)=\left(\begin{array}{cc}
i & 0 \\
0 & -i
\end{array}\right), \lambda(-1,1)=\left(\begin{array}{cc}
0 & -1 \\
1 & 0
\end{array}\right), \lambda(-1,-1)=\left(\begin{array}{cc}
0 & i \\
-i & 0
\end{array}\right) .
$$

The cocycle, with values in $\{ \pm 1\}$, is given by

$$
\lambda(\gamma) \lambda\left(\gamma^{\prime}\right)=\natural_{x}\left(\gamma, \gamma^{\prime}\right) \lambda\left(\gamma \gamma^{\prime}\right)
$$


In the twisted extended quotient $(X / / \Gamma)_{\natural}$ the fiber over $(0,0)$ is in bijection with the set of irreducible representations of

$$
\mathbb{C}\left[\Gamma_{(0,0)}, \natural_{(0,0)}\right]=\mathbb{C}[\Gamma, \emptyset] \cong M_{2}(\mathbb{C}),
$$

so this fiber consists of a single point. The quotients of $X$ by $\Gamma$ look like:

ordinary quotient (untwisted) extended quotient twisted extended quotient
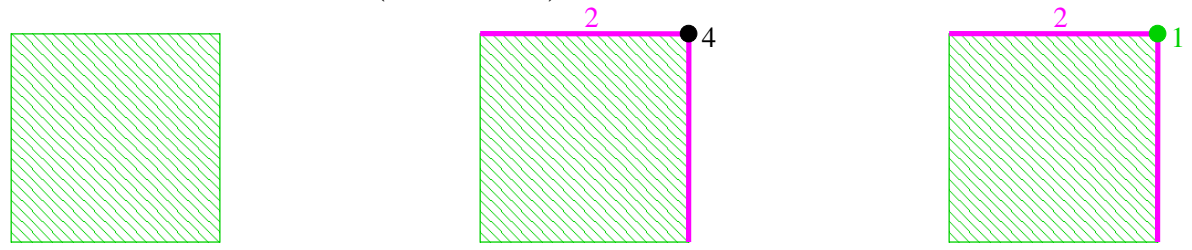

More generally, twisted extended quotients arise in the following situation. Let $A$ be a $\mathbb{C}$-algebra such that all irreducible $A$-modules have countable dimension over $\mathbb{C}$. Let $\Gamma$ be a group acting on $A$ by automorphisms and form the crossed product $A \rtimes \Gamma$.

Let $X=\operatorname{Irr}(A)$. Now $\Gamma$ acts on $\operatorname{Irr}(A)$ and we get $\downarrow$ as follows. Given $x \in \operatorname{Irr}(A)$ choose an irreducible representation $\left(\pi_{x}, V_{x}\right)$ whose isomorphism class is $x$. For each $\gamma \in \Gamma$ consider $\pi_{x}$ twisted by $\gamma$ :

$$
\gamma \cdot \pi_{x}: a \mapsto \pi_{x}\left(\gamma^{-1} a \gamma\right)
$$

Then $\gamma \cdot x$ is defined as the isomorphism class of $\gamma \cdot \pi_{x}$. Since $\gamma \cdot \pi_{x}$ is equivalent to $\pi_{\gamma x}$, there exists a nonzero intertwining operator

$$
T_{\gamma, x} \in \operatorname{Hom}_{A}\left(\gamma \cdot \pi_{x}, \pi_{\gamma x}\right) .
$$

By Schur's lemma (which is applicable because $\operatorname{dim} V_{x}$ is countable) $T_{\gamma, x}$ is unique up to scalars, but in general there is no preferred choice. For $\gamma, \gamma^{\prime} \in \Gamma_{x}$ there exists a unique $c \in \mathbb{C}^{\times}$such that

$$
c T_{\gamma, x} \circ T_{\gamma^{\prime}, x}=T_{\gamma \gamma^{\prime}, x}
$$

We define the 2-cocycle by

$$
\natural_{x}\left(\gamma, \gamma^{\prime}\right)=c .
$$

Notice the difference between (18) and (20). Let $N_{\gamma, x}$ with $\gamma \in \Gamma_{x}$ be the standard basis of $\mathbb{C}\left[\Gamma_{x}, \natural_{x}\right]$. The algebra homomorphism $\phi_{\gamma, x}$ is essentially conjugation by $T_{\gamma, x}$, but we must be careful if some of the $T_{\gamma}$ coincide. The precise definition is

$$
\phi_{\gamma, x}\left(N_{\gamma^{\prime}, x}\right)=\lambda^{-1} N_{\gamma \gamma^{\prime} \gamma^{-1}, \gamma x} \quad \text { if } \quad T_{\gamma, x} T_{\gamma^{\prime}, x} T_{\gamma, x}^{-1}=\lambda T_{\gamma \gamma^{\prime} \gamma^{-1}, \gamma x}, \lambda \in \mathbb{C}^{\times} .
$$

Suppose that $\Gamma_{x}$ is finite and $\left(\tau, V_{\tau}\right) \in \operatorname{Irr}\left(\mathbb{C}\left[\Gamma_{x}, \mathfrak{\natural}_{x}\right]\right)$. Then $V_{x} \otimes V_{\tau}$ is an irreducible $A \rtimes \Gamma_{x}$-module, where $\gamma \in \Gamma_{x}$ acts as $T_{\gamma, x} \otimes \tau\left(N_{\gamma, x}\right)$.

Lemma 2.1. [ABPS6, Lemma 2.3]

Let $A$ and $\Gamma$ be as above and assume that the action of $\Gamma$ on $\operatorname{Irr}(A)$ has finite isotropy groups.

(a) There is a bijection

$$
\begin{array}{ccc}
(\operatorname{Irr}(A) / / \Gamma)_{\natural} & \longleftrightarrow & \operatorname{Irr}(A \rtimes \Gamma) \\
\left(\pi_{x}, \tau\right) & \mapsto & \pi_{x} \rtimes \tau:=\operatorname{Ind}_{A \rtimes \Gamma}^{A \rtimes \Gamma}\left(V_{x} \otimes V_{\tau}\right) .
\end{array}
$$


(b) If all irreducible A-modules are one-dimensional, then part (a) becomes a natural bijection

$$
(\operatorname{Irr}(A) / / \Gamma)_{2} \longleftrightarrow \operatorname{Irr}(A \rtimes \Gamma)
$$

Via the following result twisted extended quotients also arise from algebras of invariants.

Lemma 2.2. Let $\Gamma$ be a finite group acting on a $\mathbb{C}$-algebra $A$. There is a bijection

$$
\begin{aligned}
\left\{V \in \operatorname{Irr}(A \rtimes \Gamma): V^{\Gamma} \neq 0\right\} & \longleftrightarrow \operatorname{Irr}\left(A^{\Gamma}\right) \\
V & \mapsto V^{\Gamma} .
\end{aligned}
$$

If all elements of $\operatorname{Irr}(A)$ have countable dimension, it becomes

$$
\begin{aligned}
& \left\{\left(\pi_{x}, \tau\right) \in(\operatorname{Irr}(A) / / \Gamma)_{\natural}:\left(V_{x} \otimes V_{\tau}\right)^{\Gamma_{x}} \neq 0\right\} \quad \longleftrightarrow \quad \operatorname{Irr}\left(A^{\Gamma}\right) \\
& \left(\pi_{x}, \tau\right) \quad \mapsto \quad\left(V_{x} \otimes V_{\tau}\right)^{\Gamma_{x}} .
\end{aligned}
$$

Proof. Consider the idempotent

$$
p_{\Gamma}=|\Gamma|^{-1} \sum_{\gamma \in \Gamma} \gamma \in \mathbb{C}[\Gamma] .
$$

It is well-known and easily shown that

$$
A^{\Gamma} \cong p_{\Gamma}(A \rtimes \Gamma) p_{\Gamma}
$$

and that the right hand side is Morita equivalent with the two-sided ideal

$$
I=(A \rtimes \Gamma) p_{\Gamma}(A \rtimes \Gamma) \subset A \rtimes \Gamma .
$$

The Morita equivalence sends a module $V$ over the latter algebra to

$$
p_{\Gamma}(A \rtimes \Gamma) \otimes_{(A \rtimes \Gamma) p_{\Gamma}(A \rtimes \Gamma)} V=V^{\Gamma} .
$$

As $I$ is a two-sided ideal,

$$
\operatorname{Irr}(I)=\{V \in \operatorname{Irr}(A \rtimes \Gamma): I \cdot V \neq 0\}=\left\{V \in \operatorname{Irr}(A \rtimes \Gamma): p_{\Gamma} V=V^{\Gamma} \neq 0\right\}
$$

This gives the first bijection. From Lemma 2.1.a we know that every such $V$ is of the form $\pi_{x} \rtimes \tau$. With Frobenius reciprocity we calculate

$$
\left(\pi_{x} \rtimes \tau\right)^{\Gamma}=\left(\operatorname{Ind}_{A \rtimes \Gamma_{x}}^{A \rtimes \Gamma}\left(V_{x} \otimes V_{\tau}\right)\right)^{\Gamma} \cong\left(V_{x} \otimes V_{\tau}\right)^{\Gamma_{x}} .
$$

Now Lemma 2.1.a and the first bijection give the second.

Let $A$ be a commutative $\mathbb{C}$-algebra all whose irreducible representations are of countable dimension over $\mathbb{C}$. Then $\operatorname{Irr}(A)$ consists of characters of $A$ and is a $T_{1^{-}}$ space. Typical examples are $A=C_{0}(X)$ (with $X$ locally compact Hausdorff), $A=$ $C^{\infty}(X)$ (with $X$ a smooth manifold) and $A=\mathcal{O}(X)$ (with $X$ an algebraic variety). As a kind of converse to Lemmas 2.1 and 2.2, we show that many twisted extended quotients of $\operatorname{Irr}(A)$ appear as the space of irreducible representations of some algebra.

Let $\Gamma$ be a finite group acting on $A$ by algebra automorphisms. Let $\tilde{\Gamma}$ be a central extension of $\Gamma$ and let $\chi_{\natural}$ be a character of $Z:=\operatorname{ker}(\tilde{\Gamma} \rightarrow \Gamma)$. For any (setwise) section $\lambda: \Gamma \rightarrow \tilde{\Gamma}$, we get a 2-cocycle $\downarrow: \Gamma \times \Gamma \rightarrow \mathbb{C}^{\times}$by

$$
\lambda(\gamma) \lambda\left(\gamma^{\prime}\right)=\natural\left(\gamma, \gamma^{\prime}\right) \lambda\left(\gamma \gamma^{\prime}\right)
$$

In fact, up to coboundaries every 2-cocycle of $\Gamma$ arises in this way [CuRe, §53]. Let

$$
p_{\natural}:=|Z|^{-1} \sum_{z \in Z} \chi_{\natural}(z)^{-1} z \quad \in \mathbb{C}[Z]
$$


be the idempotent associated to $\chi_{\mathfrak{\natural}}$. It is central in $\mathbb{C}[\tilde{\Gamma}]$ and

$$
p_{\natural} \mathbb{C}[\tilde{\Gamma}] \cong \mathbb{C}[\Gamma, \emptyset] .
$$

Lift the action of $\Gamma$ on $A$ to $\tilde{\Gamma}$ via the given projection. The algebra $A \rtimes \tilde{\Gamma}=A \rtimes \mathbb{C}[\tilde{\Gamma}]$ contains $A \rtimes p_{\natural} \mathbb{C}[\tilde{\Gamma}]$ as a direct summand.

Lemma 2.3. There is a bijection

$$
\begin{array}{ccc}
(\operatorname{Irr}(A) / / \Gamma)_{\natural} & \longleftrightarrow & \operatorname{Irr}\left(A \rtimes p_{\natural} \mathbb{C}[\tilde{\Gamma}]\right) \\
\left(\mathbb{C}_{x}, \tau\right) & \mapsto & \operatorname{Ind}_{A \rtimes \tilde{\Gamma}_{x}}^{A \rtimes \tilde{\Gamma}_{x}}\left(\mathbb{C}_{x} \otimes V_{\tau}\right) .
\end{array}
$$

Proof. Start with Lemma 2.1.b for $A$ and $\tilde{\Gamma}$. Since $Z$ acts trivially on $A$, it is contained in $\tilde{\Gamma}_{x}$ for every $x \in \operatorname{Irr}(A)$. Now restrict to representations on which $Z$ acts by $\chi_{\natural}$.

\subsection{The Bernstein decomposition.}

We return to our reductive $p$-adic group $G$. Recall that an irreducible (smooth, complex) $G$-representation is called supercuspidal if it does not appear in any $G$ representation induced from a proper Levi subgroup of $G$. Bernstein [BeDe, §2] realised that an irreducible $G$-representation is supercuspidal if and only if it is compact. Here compact means that the representation behaves like one of a compact group, in the sense that all its matrix coefficients have compact support modulo the centre of $G$. This observation enabled him to prove that the supercuspidal representations generate a direct factor of the category of smooth $G$-representations $\operatorname{Rep}(G)$.

That constitutes the first and most important step towards the Bernstein decomposition, which we describe next. Let $P$ be a parabolic subgroup of $G$ and let $L$ be a Levi factor of $P$. Let $\omega$ be a supercuspidal $L$-representation. (By definition this entails that $\omega$ is irreducible.) We call $(L, \omega)$ a cuspidal pair, and we consider such pairs up to inertial equivalence. This is the equivalence relation generated by:

- unramified twists, $(L, \omega) \sim(L, \omega \otimes \chi)$ for $\chi \in X_{\mathrm{nr}}(L)$, where $X_{\mathrm{nr}}(L)$ is the group of unramified (not necessarily unitary) characters $L \rightarrow \mathbb{C}^{\times}$;

- $G$-conjugation, $(L, \omega) \sim\left(g L g^{-1}, g \cdot \omega\right)$ for $g \in G$.

We denote a typical inertial equivalence class by $\mathfrak{s}=[L, \omega]_{G}$. In particular

$$
\mathfrak{s}_{L}:=[L, \omega]_{L}=\left\{\omega \otimes \chi \in \operatorname{Irr}(L): \chi \in X_{\mathrm{nr}}(L)\right\} .
$$

From $\mathfrak{s}$ Bernstein built a block in the category of smooth $G$-representations, in the following way. Denote the normalized parabolic induction functor by $I_{P}^{G}$. We define

$$
\begin{aligned}
& \operatorname{Irr}(G)^{\mathfrak{s}}=\left\{\pi \in \operatorname{Irr}(G): \pi \text { is a constituent of } I_{P}^{G}(\omega \otimes \chi) \text { for some } \omega \in \mathfrak{s}_{L}\right\}, \\
& \operatorname{Rep}(G)^{\mathfrak{s}}=\left\{\pi \in \operatorname{Rep}(G) \text { : every irreducible constituent of } \pi \text { belongs to } \operatorname{Irr}(G)^{\mathfrak{s}}\right\} .
\end{aligned}
$$

We denote the set of all inertial equivalence classes for $G$ by $\mathfrak{B}(G)$.

Theorem 2.4. [BeDe, Proposition 2.10]

The category of smooth $G$-representations decomposes as

$$
\operatorname{Rep}(G)=\prod_{\mathfrak{s} \in \mathfrak{B}(G)} \operatorname{Rep}(G)^{\mathfrak{s}} .
$$

The space of irreducible G-representations is a disjoint union

$$
\operatorname{Irr}(G)=\bigsqcup_{\mathfrak{s} \in \mathfrak{B}(G)} \operatorname{Irr}(G)^{\mathfrak{s}} .
$$


Let $\operatorname{Irr}_{\text {cusp }}(L)$ be the set of supercuspidal $L$-representations, up to isomorphism. For $\omega \in \operatorname{Irr}_{\text {cusp }}(L)$ (and in fact for every irreducible $L$-representation) the group

$$
X_{\mathrm{nr}}(L, \omega):=\left\{\chi \in X_{\mathrm{nr}}(L): \omega \otimes \chi \cong \omega\right\}
$$

is finite. Thus there is a bijection

$$
X_{\mathrm{nr}}(L) / X_{\mathrm{nr}}(L, \omega) \rightarrow \operatorname{Irr}(L)^{\mathfrak{s}_{L}}: \chi \mapsto \omega \otimes \chi,
$$

which endows $\operatorname{Irr}(L)^{\mathfrak{s}_{L}}$ with the structure of a complex torus. Up to isomorphism this torus depends only on $\mathfrak{s}$, and it is known as the Bernstein torus $T_{\mathfrak{s}}$. We note that $T_{\mathfrak{s}}$ is only an algebraic variety, it is not endowed with a natural multiplication map. In fact it does not even possess an unambigous "unit", because in general there is no preferred choice of an element $\omega \in \mathfrak{s}_{L}$.

Consider $W(G, L)=N_{G}(L) / L$, the "Weyl" group of $(G, L)$. It acts on $\operatorname{Irr}(L)$ by

$$
w \cdot \pi=\left[\bar{w} \cdot \pi: l \mapsto \pi\left(\bar{w}^{-} l \bar{w}\right)\right] \quad \text { for any lift } \bar{w} \in N_{G}(L) \text { of } w \in W(G, L) .
$$

To $\mathfrak{s}$ Bernstein also associated the finite group

$$
W_{\mathfrak{s}}:=\left\{w \in W(G, L): w \cdot \operatorname{Irr}(L)^{\mathfrak{s}_{L}}=\operatorname{Irr}(L)^{\mathfrak{s}_{L}}\right\} .
$$

It acts naturally on $T_{\mathfrak{s}}$, by automorphisms of algebraic varieties.

Closely related to the Bernstein decomposition is the theory of the Bernstein centre. By [BeDe, Théorème 2.13] the categorical centre of the Bernstein block $\operatorname{Rep}^{\mathfrak{s}}(G)$ is

$$
Z\left(\operatorname{Rep}(G)^{\mathfrak{s}}\right) \cong \mathcal{O}\left(T_{\mathfrak{s}}\right)^{W_{\mathfrak{s}}}=\mathcal{O}\left(T_{\mathfrak{s}} / W_{\mathfrak{s}}\right)
$$

Here $\mathcal{O}$ stands for the regular functions on an affine variety. Moreover the map

$$
\text { sc }: \operatorname{Irr}(G)^{\mathfrak{s}} \rightarrow T_{\mathfrak{s}} / W_{\mathfrak{s}}
$$

induced by (27) is surjective and has finite fibers [BeDe, §3]. Theorem 2.4 implies that every $\pi \in \operatorname{Irr}(G)$ is a constituent of $I_{P}^{G}(\omega)$, where $[L, \omega]_{G}$ is uniquely determined. By (27) the supercuspidal $L$-representation $\omega \in T_{\mathfrak{s}}$ is in fact uniquely determined up to $W_{\mathfrak{s}}$. The map $\pi \mapsto W_{\mathfrak{s}} \omega$ is just sc, and for this reason it is called the cuspidal support map. Via this map $\operatorname{Irr}^{\mathfrak{s}}(G)$ can be regarded as a non-separated algebraic variety lying over $T_{\mathfrak{s}} / W_{\mathfrak{s}}$.

\subsection{Geometric structure of Bernstein components.}

Let $\mathfrak{s}=[L, \omega]_{G}$ be an inertial equivalence class for $G$. Based on many examples, we believe that the geometric structure of the component $\operatorname{Irr}^{\mathfrak{s}}(G)$ of $\operatorname{Irr}(G)$ is related to its Bernstein centre $\mathcal{O}\left(T_{\mathfrak{s}} / W_{\mathfrak{s}}\right)$ in a strikingly simple and precise way.

Let $W_{\mathfrak{s}, t}$ be the stabilizer in $W_{\mathfrak{s}}$ of a point $t \in T_{\mathfrak{s}}$.

Conjecture 2. There exists a family of 2-cocycles

$$
\natural_{t}: W_{\mathfrak{s}, t} \times W_{\mathfrak{s}, t} \rightarrow \mathbb{C}^{\times} \quad t \in T_{\mathfrak{s}},
$$

and a bijection

such that:

$$
\operatorname{Irr}(G)^{\mathfrak{s}} \longleftrightarrow\left(T_{\mathfrak{s}} / / W_{\mathfrak{s}}\right)_{\mathfrak{\natural}}
$$

- It restricts to a bijection between tempered representations and the unitary part of the extended quotient (as explained below).

- The bijection is canonical up to permutations within L-packets. That is, for any $\phi \in \Phi(G)$, the image of $\Pi_{\phi}(G) \cap \operatorname{Irr}^{\mathfrak{s}}(G)$ is canonically defined (assuming a $L L C$ for $G$ exists). 
Let $\operatorname{Irr}_{\text {cusp }}(L)$ be the set of supercuspidal $L$-representations. It is stable under the $W(G, L)$-action (25). The definitions of $W_{\mathfrak{s}}$ and of extended quotients imply that for a fixed Levi subgroup $L$ of $G$ there is a natural bijection

$$
\bigsqcup_{\mathfrak{s}=[L, \omega]_{G}}\left(T_{\mathfrak{s}} / / W_{\mathfrak{s}}\right)_{\mathfrak{\natural}} \rightarrow\left(\operatorname{Irr}_{\text {cusp }}(L) / / W(G, L)\right)_{\mathfrak{\natural}} .
$$

In view of Theorem 2.4, Conjecture 2 can also be formulated, more elegantly, in terms of a bijection

$$
\operatorname{Irr}(G) \longleftrightarrow \bigsqcup_{L}\left(\operatorname{Irr}_{\text {cusp }}(L) / / W(G, L)\right)_{\text {七 }},
$$

where $L$ runs through a set of representatives for the $G$-conjugacy classes of Levi subgroups of $G$. In this version, our conjecture asserts that $\operatorname{Irr}(G)$ is determined by a much smaller set of data, namely the supercuspidal representations of Levi subgroups $L$ of $G$, and the actions of the Weyl groups $W(G, L)$ on those.

We expect that the group cohomology classes $\natural_{t} \in H^{2}\left(W_{\mathfrak{s}, t}, \mathbb{C}^{\times}\right)$reflect the character of $Z\left(G_{\mathrm{sc}}^{\vee}\right)^{\mathbf{W}_{F}}$ which via the Kottwitz isomorphism (12) determines how $G$ is an inner twist of a quasi-split group. In particular $\downarrow$ should be trivial whenever $G$ is quasi-split. The simplest known example of a nontrivial cocycle involves a non-split inner form of $\mathrm{SL}_{10}(F)$ [ABPS4, Example 5.5]. That example also shows that it is sometimes necessary to use twisted extended quotients in Conjecture 2.

Recall [Wal, §III.1-III.2] that a supercuspidal representation is tempered if and only if it is unitary. Let $T_{\mathfrak{s}, \text { un }}$ be the set of unitary representations in $T_{\mathfrak{s}}$, a $W_{\mathfrak{s}}$-stable compact real subtorus. Let us denote the group of unitary unramified characters of $L$ by $X_{\text {unr }}(L)$. Without loss of generality we may assume that the basepoint $\omega \in T_{\mathfrak{s}}$ is unitary. Then (24) becomes a bijection

$$
X_{\mathrm{unr}}(L) / X_{\mathrm{nr}}(L, \omega) \rightarrow T_{\mathfrak{s}, u n}: \chi \mapsto \omega \otimes \chi .
$$

Let $X_{\mathrm{nr}}^{+}(L)$ be the group of unramified characters $L \rightarrow \mathbb{R}_{>0}$. The polar decomposition of $X_{\mathrm{nr}}(L)$ reads

$$
X_{\mathrm{nr}}(L)=X_{\mathrm{unr}}(L) \times X_{\mathrm{nr}}^{+}(L) .
$$

Since $X_{\mathrm{nr}}(L, \omega)$ is finite and $\mathbb{R}_{>0}$ has no nontrivial finite subgroups, $X_{\mathrm{nr}}(L, \omega) \cap$ $X_{\mathrm{nr}}^{+}(L)=\{1\}$. Hence the canonical map

$$
T_{\mathfrak{s}, \text { un }} \times X_{\mathrm{nr}}^{+}(L) \rightarrow T_{\mathfrak{s}}:\left(\sigma, \chi^{+}\right) \mapsto \sigma \otimes \chi^{+}
$$

is bijective. We regard (31) as the polar decomposition of $T_{\mathfrak{s}}$.

Let $\operatorname{Irr}_{\text {temp }}(G)$ be the set of irreducible tempered $G$-representations (still considered up to isomorphism) and write

$$
\operatorname{Irr}_{\text {temp }}(G)^{\mathfrak{s}}=\operatorname{Irr}(G)^{\mathfrak{s}} \cap \operatorname{Irr}_{\text {temp }}(G) .
$$

Conjecture 2 asserts that there is a bijection

$$
\operatorname{Irr}_{\text {temp }}(G)^{\mathfrak{s}} \longleftrightarrow\left(T_{\mathfrak{s}, \text { un }} / / W_{\mathfrak{s}}\right)_{\mathfrak{t}} .
$$

In view of the $W_{\mathfrak{s}}$-equivariant polar decomposition $(31),\left(T_{\mathfrak{s}} / / W_{\mathfrak{s}}\right)_{\mathfrak{\natural}}$ is a natural way the complexification of its compact real form $\left(T_{\mathfrak{s}, u n} / / W_{\mathfrak{s}}\right)_{\mathfrak{t}}$. Similarly $\operatorname{Irr}^{\mathfrak{s}}(G)$ can be regarded as the "complexification" of $\operatorname{Irr}_{\text {temp }}(G)^{\mathfrak{s}}$ [ABPS1, §2]. If we manage to construct a bijection (32) with suitable properties, then the method of [ABPS1, §4] shows that it extends to a bijection $\operatorname{Irr}(G)^{\mathfrak{s}} \longleftrightarrow\left(T_{\mathfrak{s}} / / W_{\mathfrak{s}}\right)_{\mathfrak{\natural}}$ with the same properties. Thus it suffices to prove Conjecture 2 for tempered representations. 
Example. Consider $G=\mathrm{GL}_{2}(F)$ with the standard diagonal torus $T$. Let $\mathfrak{s}=\left[T, \operatorname{triv}_{T}\right]_{G}$. Then

$$
T_{\mathfrak{s}}=X_{\mathrm{nr}}(T) \cong\left(\mathbb{C}^{\times}\right)^{2}
$$

and $W_{\mathfrak{s}}=\left\{1,\left(\begin{array}{ll}0 & 1 \\ 1 & 0\end{array}\right)\right\}$, acting on $T_{\mathfrak{s}}$ by permutations of the two coordinates. In this case all the 2-cocycles $\natural_{t}$ are trivial and the extended quotient is

$$
\left(T_{\mathfrak{s}} / / W_{\mathfrak{s}}\right)_{2}=T_{\mathfrak{s}} / W_{\mathfrak{s}} \times\{\operatorname{triv}\} \sqcup\left\{\left((z, z), \operatorname{sign}_{W_{\mathfrak{s}}}\right): z \in \mathbb{C}^{\times}\right\}
$$

The bijection from Conjecture 2 is canonical:

$$
\begin{aligned}
\operatorname{Irr}(G)^{\mathfrak{s}} & \longleftrightarrow\left(T_{\mathfrak{s}} / / W_{\mathfrak{s}}\right)_{2} \\
I_{B}^{G}\left(z, z^{\prime}\right) & \longleftrightarrow\left(\left(z, z^{\prime}\right), \text { triv }\right) \\
L\left(I_{B}^{G}\left(q_{F} z, z\right)\right) & \longleftrightarrow\left(\left(q_{F} z, z\right), \text { triv }\right) \\
S t_{G} \otimes z^{\nu_{F} \text { odet }} & \longleftrightarrow\left((z, z), \operatorname{sign}_{W_{\mathfrak{s}}}\right)
\end{aligned} \quad z^{\prime} \in \mathbb{C}^{\times} \backslash\left\{q_{F} z, q_{F}^{-1} z\right\}
$$

The description of $\operatorname{Irr}\left(\mathrm{GL}_{2}(F)\right)^{\mathfrak{s}}$ is well-known, a clear account of it can be found in [BuHe, §17]. To write it down we used

$B=$ standard Borel subgroup, the upper triangular matrices in $\mathrm{GL}_{2}(F)$,

$q_{F}=\left|k_{F}\right|$, cardinality of the residue field of $F$,

$L(\pi)=$ Langlands quotient of the parabolically induced representation $\pi$,

$S t_{G}=$ Steinberg representation of $G$,

$\nu_{F} \quad=$ discrete valuation of the field $F$.

Example. Take $G=\mathrm{SL}_{2}(F)$, and the other notations as above but for $\mathrm{SL}_{2}(F)$. Now

$$
T_{\mathfrak{s}} \rightarrow \mathbb{C}^{\times}: \chi \mapsto \chi\left(\left(\begin{array}{cc}
\varpi_{F} & 0 \\
0 & \varpi_{F}^{-1}
\end{array}\right)\right)
$$

is a bijection, for any uniformizer $\varpi_{F}$ of $F$. The group $W_{\mathfrak{s}}=\{1, w\}$ acts on $T_{\mathfrak{s}}$ by $w \cdot z=z^{-1}$. The relevant extended quotient is

$$
\left(T_{\mathfrak{s}} / / W_{\mathfrak{s}}\right)_{2}=T_{\mathfrak{s}} / W_{\mathfrak{s}} \times\{\operatorname{triv}\} \sqcup\left\{\left( \pm 1, \operatorname{sign}_{W_{\mathfrak{s}}}\right)\right\}
$$

It is in bijection with $\operatorname{Irr}(G)^{\mathfrak{s}}$ via

$$
\begin{array}{rlrl}
\operatorname{Irr}(G)^{\mathfrak{s}} & \longleftrightarrow & \left(T_{\mathfrak{s}} / / W_{\mathfrak{s}}\right)_{2} & \\
I_{B}^{G}(z) & \longleftrightarrow & (z, \text { triv }) & z \in \mathbb{C}^{\times} \backslash\left\{-1, q_{F}, q_{F}^{-1}\right\} \\
L\left(I_{B}^{G}\left(q_{F}\right)\right) & \longleftrightarrow & \left(q_{F}, \text { triv }\right) & \\
S t_{G} & \longleftrightarrow & \left(1, \operatorname{sign}_{W_{\mathfrak{s}}}\right) & \\
I_{B}^{G}(-1)=\pi_{+} \oplus \pi_{-} & \longleftrightarrow\left\{\left(-1, \operatorname{triv}_{W_{\mathfrak{s}}}\right),\left(-1, \operatorname{sign}_{W_{\mathfrak{s}}}\right)\right\} &
\end{array}
$$

Notice that the unramified character $\left(\begin{array}{ll}a & 0 \\ 0 & a^{-1}\end{array}\right) \mapsto(-1)^{\nu_{F}(a)}$ gives rise to an L-packet with two irreducible $G$-representations, denoted $\pi_{ \pm}$. Both must be mapped to a point in the extended quotient, lying over $-1 \in T_{\mathfrak{s}} / W_{\mathfrak{s}}$. There are two ways to do so, both equally good. There does not seem to be a canonical choice without specifying additional data, see [ABPS6, Example 11.3].

At the time of writing, Conjecture 2 has been proven in the following cases.

- General linear groups over division algebras [ABPS4, ABPS7].

- Special linear groups over division algebras [ABPS4, ABPS7].

- Split orthogonal and symplectic groups [Mou, §5].

- Principal series representations of split groups [ABPS5], [ABPS6, §18-19]. 


\subsection{Hecke algebras for Bernstein blocks.}

We will explain some of the ideas that lead to the proof of Conjecture 2 in the aforementioned cases. Let $\mathcal{H}(G)$ be the Hecke algebra of $G$, that is, the vector space $C_{c}^{\infty}(G)$ of locally constant compactly supported functions on $G$, endowed with the convolution product. It is the version of the group algebra of $G$ which is most suitable for studying smooth representations. The category $\operatorname{Rep}(G)$ is naturally equivalent with the category $\operatorname{Rep}(\mathcal{H}(G)$ ) of $\mathcal{H}(G)$-modules $V$ such that $\mathcal{H}(G) \cdot V=V$. (The latter condition is nontrivial because $\mathcal{H}(G)$ does not have a unit if $G \neq 1$.)

In these terms the Bernstein decomposition becomes

$$
\begin{aligned}
& \mathcal{H}(G)=\bigoplus_{\mathfrak{s} \in \mathfrak{B}(G)} \mathcal{H}(G)^{\mathfrak{s}}, \\
& \operatorname{Rep}(G) \cong \bigoplus_{\mathfrak{s} \in \mathfrak{B}(G)} \operatorname{Rep}\left(\mathcal{H}(G)^{\mathfrak{s}}\right), \\
& \operatorname{Irr}(G)=\bigsqcup_{\mathfrak{s} \in \mathfrak{B}(G)} \operatorname{Irr}\left(\mathcal{H}(G)^{\mathfrak{s}}\right) .
\end{aligned}
$$

In other words, $\operatorname{Rep}\left(\mathcal{H}(G)^{\mathfrak{s}}\right)$ is a Bernstein block for $G$. Unfortunately, the algebras $\mathcal{H}(G)^{\mathfrak{s}}$ are in general too large to work well with. To perform interesting computations, one has to downsize them. The most common approach is due to Bushnell and Kutzko [BuKu1, BuKu2]. They propose to look for suitable idempotents $e_{\mathfrak{s}} \in \mathcal{H}(G)$ such that:

- $\mathcal{H}(G)^{\mathfrak{s}}=\mathcal{H}(G) e_{\mathfrak{s}} \mathcal{H}(G)$, and this is Morita equivalent with $e_{\mathfrak{s}} \mathcal{H}(G) e_{\mathfrak{s}}$ via the $\operatorname{map} V \mapsto e_{\mathfrak{s}} V$;

- $e_{\mathfrak{s}} \mathcal{H}(G) e_{\mathfrak{s}}$ is smaller and simpler than $\mathcal{H}(G)^{\mathfrak{s}}$.

Typically $e_{\mathfrak{s}}$ will be associated to an irreducible representation of a compact open subgroup of $G$, then Bushnell and Kutzko call it a type for $\mathfrak{s}$. Yet in some cases this might be asking for too much, so we rather not require that.

The challenge is to find an idempotent such that the structure of $e_{\mathfrak{s}} \mathcal{H}(G) e_{\mathfrak{s}}$ is nice and explicit. Let us call such an $e_{\mathfrak{s}}$ a nice idempotent for $\mathfrak{s}$. In practice this means that $e_{\mathfrak{s}} \mathcal{H}(G) e_{\mathfrak{s}}$ must be close to an affine Hecke algebra. Such algebras can be defined in several ways [IwMa, Lus4], here we present a construction which is welladapted to representations of $p$-adic groups. Let $T$ a complex torus with character lattice $X^{*}(T)$. Let $R \subset X^{*}(T)$ be a root system, not necessarily reduced. The Weyl group $W(R)$ acts on $T, X^{*}(T), \mathcal{O}(T)$ and $R$. We also need a parameter function $q: R / W(R) \rightarrow \mathbb{R}_{>0}$.

Definition 2.5. The affine Hecke algebra $\mathcal{H}(T, R, q)$ is the $\mathbb{C}$-algebra such that:

- As vector space it equals $\mathcal{O}(T) \otimes \mathbb{C}[W(R)]$.

- $\mathcal{O}(T)$ is embedded as a subalgebra.

- $\mathbb{C}[W(R)]=\operatorname{span}\left\{N_{w}: w \in W(R)\right\}$ is embedded as the Iwahori-Hecke algebra $\mathcal{H}(W(R), q)$, that is, the multiplication is defined by

$$
\begin{array}{ll}
N_{w} N_{v}=N_{w v} & \text { if } \ell(w)+\ell(v)=\ell(w v), \\
\left(N_{s_{\alpha}}-q_{\alpha}^{1 / 2}\right)\left(N_{s_{\alpha}}+q_{\alpha}^{-1 / 2}\right)=0 & \text { for every simple reflection } s_{\alpha} .
\end{array}
$$

Here $\ell$ is the length function of $W(R)$ and $\alpha \in R$ is a simple root.

- The commutation rules between $\mathcal{O}(T)$ and $\mathcal{H}(W(R), q)$ are determined by

$$
f N_{s_{\alpha}}-N_{s_{\alpha}} s_{\alpha}(f)=\left(q_{\alpha}^{1 / 2}-q_{\alpha}^{-1 / 2}\right) \frac{f-s_{\alpha}(f)}{1-\theta_{-\alpha}} .
$$

Here $f \in \mathcal{O}(T), \alpha$ is a simple root and $\theta_{x} \in \mathcal{O}(T)$ corresponds to $x \in X^{*}(T)$. (In fact the formula can be slightly more complicated if $R$ contains a factor of type $C_{l}$, see [Lus4, §3].) 
Notice that for the parameter function $q=1$ we get

$$
\mathcal{H}(T, R, 1)=\mathcal{O}(T) \rtimes W(R)=\mathbb{C}\left[X^{*}(T) \rtimes W(R)\right] .
$$

With Lemma 2.1.b we obtain a natural bijection

$$
\operatorname{Irr}(\mathcal{O}(T) \rtimes W(R)) \longleftrightarrow(T / / W(R))_{2} .
$$

The representations of affine Hecke algebras have been subjected to a lot of study, see in particular [Lus4, KaLu, Opd, Sol2]. As a result the representation theory of $\mathcal{H}(T, R, q)$ is understood quite well, and close relations between $\operatorname{Irr}(\mathcal{H}(T, R, q))$ and $\operatorname{Irr}(\mathcal{H}(T, R, 1)) \cong(T / / W(R))_{2}$ are known. This is the main source of extended quotients in the representation theory of reductive $p$-adic groups.

Now we provide an overview of what is known about the structure of $e_{\mathfrak{s}} \mathcal{H}(G) e_{\mathfrak{s}}$ in various cases.

\section{Iwahori-spherical representations.}

This is the classical case. Let $M$ be a minimal Levi subgroup of $G$ and $\mathfrak{s}=$ $\left[M, \operatorname{triv}_{M}\right]_{G}$. Borel [Bor1] showed that the idempotent $e_{I}$ associated to an Iwahori subgroup $I$ is nice for $\mathfrak{s}$. By [IwMa, §3] there is an algebra isomorphism

$$
C_{c}(I \backslash G / I) \cong e_{I} \mathcal{H}(G) e_{I} \cong \mathcal{H}\left(X_{\mathrm{nr}}(M), R^{\vee}(G, M), q_{I}\right),
$$

where $R^{\vee}(G, M)$ is the system of coroots of $G$ with respect to the maximal split torus in $Z(M)$ and $q_{I, \alpha}=\operatorname{vol}\left(I s_{\alpha} I\right) / \operatorname{vol}(I)$ for a simple reflection $s_{\alpha}$.

\section{Principal series representations of split groups.}

Suppose that $G$ is $F$-split and let $T$ be a maximal split torus of $G$. Fix a smooth character $\chi_{\mathfrak{s}} \in \operatorname{Irr}(T)$ and put $\mathfrak{s}=\left[T, \chi_{\mathfrak{s}}\right]_{G}$, so that

$$
X_{\mathrm{nr}}(T) \rightarrow T_{\mathfrak{s}}: \chi \mapsto \chi \chi_{\mathfrak{s}}
$$

is a homeomorphism. By [Roc, Lemma 6.2] there exist a root subsystem $R_{\mathfrak{s}} \subset$ $R^{\vee}(G, T)$ and a subgroup $\mathfrak{R}_{\mathfrak{s}} \subset W_{\mathfrak{s}}$ such that $W_{\mathfrak{s}}=W\left(R_{\mathfrak{s}}\right) \rtimes \mathfrak{R}_{\mathfrak{s}}$.

Theorem 2.6. [Roc, Theorem 6.3]

There exists a type for $\mathfrak{s}$ and an algebra isomorphism

$$
e_{\mathfrak{s}} \mathcal{H}(G) e_{\mathfrak{s}} \cong \mathcal{H}\left(T_{\mathfrak{s}}, R_{\mathfrak{s}}, q\right) \rtimes \mathfrak{R}_{\mathfrak{s}},
$$

where $q_{\alpha}=\left|k_{F}\right|$ for all $\alpha \in R_{\mathfrak{s}}$.

\section{Level zero representations.}

These are $G$-representations which contain non-zero vectors fixed by the pro-unipotent radical of a parahoric subgroup of $G$. For such representations the algebra $e_{\mathfrak{s}} \mathcal{H}(G) e_{\mathfrak{s}}$ can be determined via suitable reductive groups over the residue field $k_{F}$.Mor, Theorem 7.12], see also [Lus5]. It turns out that, like Theorem 2.6, $e_{\mathfrak{s}} \mathcal{H}(G) e_{\mathfrak{s}}$ is of the form $\mathcal{H}\left(T_{\mathfrak{s}}, R_{\mathfrak{s}}, q_{\mathfrak{s}}\right) \rtimes \mathbb{C}\left[\mathfrak{R}_{\mathfrak{s}}, h_{\mathfrak{s}}\right]$ for suitable $R_{\mathfrak{s}}, q_{\mathfrak{s}}$ and $\mathfrak{R}_{\mathfrak{s}}$. In all examples of level zero Bernstein blocks which have been worked out, the 2 -cocycle $\mathfrak{h}_{\mathfrak{s}}$ of $\mathfrak{R}_{\mathfrak{s}}$ is trivial.

\section{Symplectic and orthogonal groups.}

For any inertial equivalence class $\mathfrak{s} \in \mathfrak{B}(G)$ Heiermann [Hei] proved that $\mathcal{H}(G)^{\mathfrak{s}}$ is Morita equivalent with $\mathcal{H}\left(T_{\mathfrak{s}}, R_{\mathfrak{s}}, q_{\mathfrak{s}}\right) \rtimes \mathfrak{R}_{\mathfrak{s}}$, for suitable $R_{\mathfrak{s}}, q_{\mathfrak{s}}$ and $\mathfrak{R}_{\mathfrak{s}}$. A type for $\mathfrak{s}$ was constructed in [MiSt]. It seems plausible that $e_{\mathfrak{s}} \mathcal{H}(G) e_{\mathfrak{s}} \cong \mathcal{H}\left(T_{\mathfrak{s}}, R_{\mathfrak{s}}, q_{\mathfrak{s}}\right) \rtimes \mathfrak{R}_{\mathfrak{s}}$, but as far as we know this has not yet been checked. 
Inner forms of $\mathrm{GL}_{n}(F)$.

Let $D$ be a division algebra with centre $F$. Every Levi subgroup of $G=\mathrm{GL}_{m}(D)$ is of the form $L=\prod_{i} \mathrm{GL}_{m_{i}}(D)^{e_{i}}$, where $\sum_{i} m_{i} e_{i}=m$. Fix $\omega \in \operatorname{Irr}_{\text {cusp }}(L)$, of the form $\omega=\bigotimes_{i=1}^{k} \omega_{i}^{\otimes e_{i}}$, where $\omega_{i} \in \operatorname{Irr}_{\text {cusp }}\left(\operatorname{GL}_{m_{i}}(D)\right)$ is not inertially equivalent with $\omega_{j}$ if $i \neq j$. Then $T_{\mathfrak{s}} \cong \prod_{i=1}^{k}\left(\mathbb{C}^{\times}\right)^{e_{i}}, R_{\mathfrak{s}}$ is of type $\prod_{i=1}^{k} A_{e_{i}-1}$ and

$$
W_{\mathfrak{s}}=W\left(R_{\mathfrak{s}}\right) \cong \prod_{i=1}^{k} S_{e_{i}}
$$

where $W_{\mathfrak{s}}$ is the group defined in (26).

Theorem 2.7. [Séc, SéSt]

There exist a type for $\mathfrak{s}$, a finite dimensional vector space $V$ and a parameter function $q_{\mathfrak{s}}: R_{\mathfrak{s}} \rightarrow q^{\mathbb{N}}$ such that

$$
e_{\mathfrak{s}} \mathcal{H}(G) e_{\mathfrak{s}} \cong \mathcal{H}\left(T_{\mathfrak{s}}, R_{\mathfrak{s}}, q_{\mathfrak{s}}\right) \otimes \operatorname{End}_{\mathbb{C}}(V) .
$$

Inner forms of $\mathrm{SL}_{n}(F)$.

Let $G=\mathrm{SL}_{m}(D)$, the kernel of the reduced norm map $\mathrm{GL}_{m}(D) \rightarrow F^{\times}$. Every Levi subgroup of $G$ looks like $L=L^{\prime} \cap \mathrm{SL}_{m}(D)$, where $L^{\prime}=\prod_{i} \mathrm{GL}_{m_{i}}(D)^{e_{i}}$. Fix $\mathfrak{s}=[L, \omega]_{G}$ and choose an $\omega^{\prime} \in \operatorname{Irr}_{\text {cusp }}\left(L^{\prime}\right)$ which contains $\omega$. Then $R_{\mathfrak{s}}$ is, as above for $\omega^{\prime}$, of type $\prod_{i=1}^{k} A_{e_{i}-1}$, but $T_{\mathfrak{s}}$ and $W_{\mathfrak{s}}$ are modified compared to $\mathrm{GL}_{m}(D)$. An explicit description of $T_{\mathfrak{s}}$ may be found in [ABPS7, Prop. 2.1].

Write $M^{\prime}=\prod_{i} \mathrm{GL}_{e_{i} m_{i}}(D)$ and let $P^{\prime}$ be the parabolic subgroup of $\mathrm{GL}_{m}(D)$ generated by $L^{\prime}$ and the upper triangular-block matrices. Then

$$
W_{\mathfrak{s}}=W\left(R_{\mathfrak{s}}\right) \rtimes \mathfrak{R}_{\mathfrak{s}} \cong \prod_{i=1}^{k} S_{e_{i}} \rtimes \mathfrak{R}_{\mathfrak{s}},
$$

with $\mathfrak{R}_{\mathfrak{s}}=W_{\mathfrak{s}} \cap N_{\mathrm{GL}_{m}(D)}\left(P^{\prime} \cap M^{\prime}\right) / L^{\prime}$.

Theorem 2.8. [ABPS4, §4.4]

There exist a finite dimensional projective representation $V$ of $X_{\mathrm{nr}}(L, \omega) \rtimes \mathfrak{R}_{\mathfrak{s}}$ and a nice idempotent $e_{\mathfrak{s}}$ for $\mathfrak{s}$, such that

$$
e_{\mathfrak{s}} \mathcal{H}(G) e_{\mathfrak{s}} \cong\left(\mathcal{H}\left(X_{\mathrm{nr}}(L), R_{\mathfrak{s}}, q_{\mathfrak{s}}\right) \otimes \operatorname{End}_{\mathbb{C}}(V)\right)^{X_{\mathrm{nr}}(L, \omega)} \rtimes \mathfrak{R}_{\mathfrak{s}} .
$$

Here $X_{\mathrm{nr}}(L, \omega) \rtimes \Re_{\mathfrak{s}}$ acts both on $\mathcal{H}\left(X_{\mathrm{nr}}(L), R_{\mathfrak{s}}, q_{\mathfrak{s}}\right)$ and on $\operatorname{End}_{\mathbb{C}}(V)$.

The algebras appearing in Theorem 2.8 are quite a bit more general than the previous ones. See $[$ ABPS4, §5] for some examples of what can happen.

For instance, they need not be Morita equivalent to an affine Hecke algebra extended by a finite group of automorphisms of the root system. That can already happen in the split case $G=\mathrm{SL}_{n}(F)$ [GoRo, $\left.\S 11.8\right]$. Moreover, the projective action of $\mathfrak{R}_{\mathfrak{s}}$ on $V$ gives rise to a possibly nontrivial 2-cocycle of $\mathfrak{R}_{\mathfrak{s}}$. It is related to the character of $Z\left(\mathrm{SL}_{n}(\mathbb{C})\right)=Z\left(G_{\mathrm{sc}}^{\vee}\right) \mathbf{W}_{F}$ that specifies $G$ as an inner twist of $\operatorname{SL}_{n}(F)$, see [ABPS4, Theorem 4.15].

From a more general point of view, the algebra in Theorem 2.8 rather closely resembles the shape of the Fourier transform of a component in the Schwartz algebra of any reductive $p$-adic group $G$ [Wal]. The main difference is that for the Schwartz algebra one has to replace $\mathcal{O}\left(T_{\mathfrak{s}}\right)$ by $C^{\infty}\left(T_{\mathfrak{s}, \text { un }}\right)$.

From $\mathfrak{s}=[L, \omega]_{G}, T_{\mathfrak{s}}$ and $W_{\mathfrak{s}}$ one can canonically deduce a root system $R_{\mathfrak{s}}$, namely the set of roots of $\left(G, Z(L)^{\circ}\right)$ for which the Harish-Chandra $\mu$-function has a pole 
on $T_{\mathfrak{s}}[\mathrm{H}-\mathrm{C}]$. The group $W_{\mathfrak{s}}$ acts on the Weyl chambers for $R_{\mathfrak{s}}$, and the stabilizer of a fixed positive chamber is a subgroup $\mathfrak{R}_{\mathfrak{s}} \subset W_{\mathfrak{s}}$. Since $W\left(R_{\mathfrak{s}}\right)$ acts simply transitively on the collection of Weyl chambers, $W_{\mathfrak{s}}=W\left(R_{\mathfrak{s}}\right) \rtimes \mathfrak{R}_{\mathfrak{s}}$. On the basis of the above, we expect:

Conjecture 3. Let $\mathfrak{s}=[L, \omega]_{G}$ be any inertial equivalence class and use the above notations. There exist a parameter function $q_{\mathfrak{s}}: R_{\mathfrak{s}} \rightarrow \mathbb{R}_{>0}$, a finite dimensional projective representation $V_{\mathfrak{s}}$ of $X_{\mathrm{nr}}(L, \omega) \rtimes \mathfrak{R}_{\mathfrak{s}}$, and a nice idempotent $e_{\mathfrak{s}}$ for $\mathfrak{s}$ such that

$$
e_{\mathfrak{s}} \mathcal{H}(G) e_{\mathfrak{s}} \cong\left(\mathcal{H}\left(X_{\mathrm{nr}}(L), R_{\mathfrak{s}}, q_{\mathfrak{s}}\right) \otimes \operatorname{End}_{\mathbb{C}}\left(V_{\mathfrak{s}}\right)\right)^{X_{\mathrm{nr}}(L, \omega)} \rtimes \mathfrak{R}_{\mathfrak{s}} .
$$

\subsection{Conjectural construction of the bijection.}

Let us return to Conjecture 2. Whenever Conjecture 3 holds for $\mathfrak{s}$, one can apply $\left[\right.$ Sol2, §5.4]. This proves an earlier version of Conjecture 2 for $\operatorname{Irr}^{\mathfrak{5}}(G)$ (formulated in terms of an extended quotient of the first kind, see [ABPS2]). To obtain Conjecture 2 completely more work is required, which has been carried out in the cases listed on page 19.

Based on knowledge of the representation theory of affine Hecke algebras and assuming Conjecture 3, we sketch how the bijection $\operatorname{Irr}(G)^{\mathfrak{s}} \rightarrow\left(T_{\mathfrak{s}} / / W_{\mathfrak{s}}\right)_{\mathfrak{\natural}}$ should be constructed. That is, we describe how the construction goes in the aforementioned known cases, and we expect that something similar works in general.

As discussed around (32) it suffices to construct

$$
\operatorname{Irr}_{\text {temp }}(G)^{\mathfrak{s}} \rightarrow\left(T_{\mathfrak{s}, \text { un }} / / W_{\mathfrak{s}}\right)_{\mathfrak{t}} \text {. }
$$

Let $\left(\pi, V_{\pi}\right) \in \operatorname{Irr}_{\text {temp }}(G)^{\mathfrak{s}}$.

- As we saw in (28), the cuspidal support of $\pi$ is an element $\mathbf{s c}(\pi) \in T_{\mathfrak{s}} / W_{\mathfrak{s}}$. Choose a lift $\mathbf{s c}(\pi) \in T_{\mathfrak{s}}$ and let $t=\mathbf{s c}(\pi)_{\text {un }} \in T_{\mathfrak{s}, \text { un }}$ be its unitary part, obtained from the polar decomposition (31). This $t$ will be the $T_{\mathfrak{s}}$-coordinate in the extended quotient.

- Let $e_{\mathfrak{s}}$ be as in Conjecture 3 , so $e_{\mathfrak{s}} V_{\pi} \in \operatorname{Rep}\left(e_{\mathfrak{s}} \mathcal{H}(G) e_{\mathfrak{s}}\right)$. Recall from (27) that

$$
Z\left(e_{\mathfrak{s}} \mathcal{H}(G) e_{\mathfrak{s}}\right) \cong Z\left(\operatorname{Rep}(G)^{\mathfrak{s}}\right) \cong \mathcal{O}\left(T_{\mathfrak{s}} / W_{\mathfrak{s}}\right)
$$

The algebra $e_{\mathfrak{s}} \mathcal{H}(G) e_{\mathfrak{s}}$ contains $\mathcal{O}\left(T_{\mathfrak{s}}\right)$ as a subalgebra such that $Z\left(e_{\mathfrak{s}} \mathcal{H}(G) e_{\mathfrak{s}}\right)=$ $\mathcal{O}\left(T_{\mathfrak{s}}\right)^{W_{\mathfrak{s}}}$. All the weights for the action of $\mathcal{O}\left(T_{\mathfrak{s}}\right)$ on $e_{\mathfrak{s}} V_{\pi}$ are contained in $W_{\mathfrak{s}} \mathbf{s c}(\pi)$, which is a subset of $W_{\mathfrak{s}} t X_{\mathrm{nr}}^{+}(L)$. As vector spaces

$$
e_{\mathfrak{s}} V_{\pi}=\bigoplus_{w \in W_{\mathfrak{s}} / W_{\mathfrak{s}, t}}\left(e_{\mathfrak{s}} V_{\pi}\right)_{w t},
$$

where $\left(e_{\mathfrak{s}} V_{\pi}\right)_{w t}$ is the linear subspace of $e_{\mathfrak{s}} V_{\pi}$ on which $\mathcal{O}\left(T_{\mathfrak{s}}\right)$ acts by weights from $w t X_{\mathrm{nr}}^{+}(L)$.

- With involved techniques from affine Hecke algebras [Lus4, Sol2] one can endow $\left(e_{\mathfrak{s}} V_{\pi}\right)_{t}$ with a linear action of $W\left(R_{\mathfrak{s}}\right)_{t}$, the stabilizer of $t$ in $W\left(R_{\mathfrak{s}}\right)$. It extends to a representation of $\mathbb{C}\left[W_{\mathfrak{s}, t}, h_{\mathfrak{s}}\right]$, where the 2 -cocycle $\mathfrak{h}_{\mathfrak{s}}$ is determined by the projective $\mathfrak{R}_{\mathfrak{s}}$-representation $V_{\mathfrak{s}}$ from Conjecture 3 . Define $\downarrow$ such that $\natural_{t}=\left.\natural_{\mathfrak{s}}\right|_{W_{\mathfrak{s}, t}}$.

- It remains to specify an irreducible representation of $\mathbb{C}\left[W_{\mathfrak{s}, t}, \mathfrak{h}_{\mathfrak{s}}\right]$, depending on $\left(e_{\mathfrak{s}} V_{\pi}\right)_{t}$. There are a root subsystem $R_{\mathfrak{s}, t}$ and a Weyl subgroup $W\left(R_{\mathfrak{s}, t}\right) \subset W\left(R_{\mathfrak{s}}\right)$. The Springer correspondence associates to every irreducible $W\left(R_{\mathfrak{s}, t}\right)$-representation a unipotent orbit in some complex reductive group. The dimension of this orbit can be regarded as an invariant, which 
we call the $a$-weight of the representation, where $a$ is the function defined by Lusztig in [Lus3]. Let $m$ be the maximal $a$-weight appearing among the $W\left(R_{\mathfrak{s}, t}\right)$-subrepresentations of $\left(e_{\mathfrak{s}} V_{\pi}\right)_{t}$, and let $V_{\rho}$ be the sum of the $W\left(R_{\mathfrak{s}, t}\right)$ subrepresentations of $a$-weight $m$. It turns out that $\left(\rho, V_{\rho}\right)$ is an irreducible $\mathbb{C}\left[W_{\mathfrak{s}, t}, h_{\mathfrak{s}}\right]$-representation.

Then (36) sends $\pi \in \operatorname{Irr}_{\text {temp }}(G)^{\mathfrak{s}}$ to $(t, \rho) \in\left(T_{\mathfrak{s}, \text { un }} / / W_{\mathfrak{s}}\right)_{\mathfrak{t}}$.

Obviously the construction of $\rho$ is very complicated, and it is hard to see just from the above sketch what is going on. We want to make the point that Conjecture 2 is not about some mysterious bijection, but about a map which we already know quite well.

Our construction also reveals some (conjectural) information about L-packets. Let $G_{\mathfrak{s}, t}^{\vee}$ be (possibly disconnected) complex reductive group with maximal torus $T_{\mathfrak{s}}$, root system $R_{\mathfrak{s}, t}$ and Weyl group $W_{\mathfrak{s}, t}$. The extension to $W_{s, t}$ of the Springer correspondence for $W\left(R_{s, t}\right)$, as in [ABPS6, Theorem 4.4], associates to $\left(\rho, V_{\rho}\right)$ a unique unipotent class $u(\rho)$ in $G_{\mathfrak{s}, t}^{\vee}$. It still depends canonically on $\pi$, because the $W\left(R_{\mathfrak{s}, t}\right)$-representation $\left(e_{\mathfrak{s}} V_{\pi}\right)_{t}$ does. Only the extension of $\left(e_{\mathfrak{s}} V_{\pi}\right)_{t}$ to a $\mathbb{C}\left[W_{\mathfrak{s}, t}, \mathfrak{h}_{\mathfrak{s}}\right]$ representation need not be canonical.

In all examples the L-parameter of $\pi$ depends only on $(t, u(\rho))$, and $\pi^{\prime} \in \operatorname{Irr}_{\text {temp }}(G)^{\mathfrak{s}}$ has the same L-parameter if and only if $W_{\mathfrak{s}}(t, u(\rho))=W_{\mathfrak{s}}\left(t^{\prime}, u\left(\rho^{\prime}\right)\right)$. Therefore we believe that the bijection in Conjecture 2 is canonical up to permutations within L-packets.

\section{Reduction to the Supercuspidal CASE}

We discuss a strategy to reduce the construction of a LLC for irreducible smooth representations to the case of supercuspidal representations. In view of the work of V. Lafforgue [Laf2, Laf3], this could be useful in large generality. (While this paper was under review, the material in this section has been worked out in [AMS].)

If one assumes the bijective LLC (Conjecture 1) for $G$ (considered as in inner twist of a quasi-split group), then the Bernstein decomposition of $\operatorname{Irr}(G)$ can be transferred to enhanced L-parameters:

$$
\Phi_{e}(G)=\bigsqcup_{\mathfrak{s} \in \mathfrak{B}(G)} \Phi_{e}(G)^{\mathfrak{s}},
$$

where $\Phi_{e}(G)^{\mathfrak{s}}$ is the set that parametrizes $\operatorname{Irr}(G)^{\mathfrak{s}}$. Fixing a character $\zeta_{G}$ of $Z\left(G_{\mathrm{sc}}^{\vee}\right)$ as in Paragraph 1.5, we obtain a similar decomposition of $\Phi_{e, \zeta_{G}}(G)$.

If we also assume Conjecture 2 for $\mathfrak{s}=[L, \omega]_{G}$, then $\operatorname{Irr}(G)^{\mathfrak{s}}$ is in bijection with a twisted extended quotient $\left(T_{\mathfrak{s}} / / W_{\mathfrak{s}}\right)_{\mathfrak{t}}$. By the conjectural LLC for supercuspidal representations of $L, T_{\mathfrak{s}}$ should be in bijection with

$$
\Phi_{e, \zeta_{G}}(L)^{\mathfrak{s}_{L}}:=\left\{(\phi, \rho) \in \Phi_{e}(L)^{\mathfrak{s}_{L}}:\left.\rho\right|_{Z\left(L_{\mathrm{sc}}^{\vee}\right)}=\left.\zeta_{G}\right|_{Z\left(L_{\mathrm{sc}}^{\vee}\right)}\right\}
$$

With the fifth desideratum of the LLC for $G$ and $L$, we get bijections

$$
\Phi_{e, \zeta_{G}}(G)^{\mathfrak{s}} \longleftrightarrow \operatorname{Irr}(G)^{\mathfrak{s}} \longleftrightarrow\left(T_{\mathfrak{s}} / / W_{\mathfrak{s}}\right)_{\mathfrak{\natural}} \longleftrightarrow\left(\Phi_{e, \zeta_{G}}(L)^{\mathfrak{s} L} / / W_{\mathfrak{s}}\right)_{\mathfrak{\natural}}
$$

If we can do this for all inertial equivalence classes $\mathfrak{s} \in \mathfrak{B}(G)$, we even obtain a bijection

$$
\Phi_{e, \zeta_{G}}(G) \longleftrightarrow \bigsqcup_{[L, \omega]_{G}=\mathfrak{s} \in \mathfrak{B}(G)}\left(\Phi_{e, \zeta_{G}}(L)^{\mathfrak{s}_{L}} / / W_{\mathfrak{s}}\right)_{\mathfrak{\natural}}
$$


Let $\Phi_{\text {cusp }}(L)$ be the subset of $\Phi_{e}(L)$ which corresponds to $\operatorname{Irr}_{\text {cusp }}(L)$. Again, its definition depends on Conjecture 1. The same argument as above can also be applied to the equivalent formulation (30) of Conjecture 2. That leads to a bijection

$$
\Phi_{e, \zeta_{G}}(G) \longleftrightarrow \bigsqcup_{L}\left(\Phi_{\text {cusp }, \zeta_{G}}(L) / / W(G, L)\right)_{\text {七 }}
$$

where $L$ runs over the conjugacy classes of Levi subgroups of $G$.

In the upcoming paragraphs we will explain how to reformulate (37) and (38) entirely in terms of complex reductive groups with Galois actions, resulting in Conjecture 4 . That and Conjecture 2 should form the vertical maps in a commutative, bijective diagram

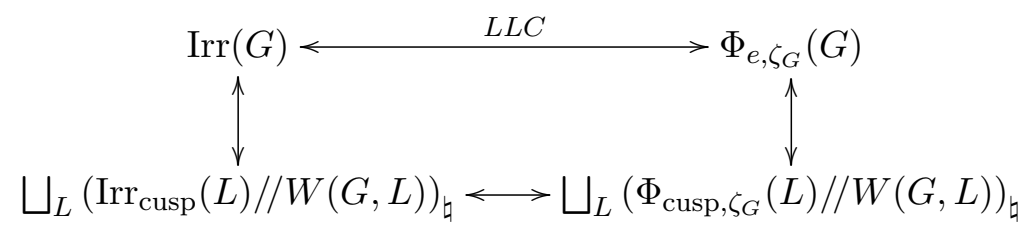

where both unions run over the same set of represenatives for the conjugacy classes of Levi subgroups of $G$. The bottom map comes from the LLC for supercuspidal $L$-representations, taking desideratum (5) and Proposition 3.1 into account. With such a diagram one can try to establish the local Langlands correspondence for $G$. This setup reduces the problem to three more manageable steps:

- Conjecture 2,

- Conjecture 4,

- the LLC for supercuspidal representations.

We note that this strategy was already employed to find the LLC for principal series representations of split reductive $p$-adic groups [ABPS6, $\S 16]$. In that case the bottom line of the above diagram is a consequence of the naturality of the LLC for (split) tori.

\subsection{Towards a Galois analogue of the Bernstein theory.}

We would like to rephrase (37) and (38) entirely on the Galois side. To get started, one has to be able to detect when an enhanced L-parameter is "cuspidal", without knowing the LLC. We note that it is impossible to define this properly for L-parameters, since there are L-packets that contain both supercuspidal and nonsupercuspidal representations. The enhancement of a L-parameter is essential for its nature.

In view of [Mou, Définition 4.11], the correct criterion should be that an enhanced L-parameter $(\phi, \rho) \in \Phi_{e}(G)$ is cuspidal if:

- $\phi \in \Phi(G)$ is discrete;

- $\rho \in \operatorname{Irr}\left(\mathcal{S}_{\phi}\right)$ is cuspidal in the sense of Lusztig's generalized Springer correspondence [Lus2].

Let $\Phi_{\text {cusp }}(G)$ denote the set of cuspidal (enhanced) L-parameters for $G$.

Furthermore a notion of "cuspidal support" of enhanced L-parameters seems necessary, that is, a well-defined map from $\Phi_{e}(G)$ to cuspidal enhanced Langlands parameters of Levi subgroups of $G$. Such a notion was developed in [Mou, §4.2.2], and worked out completely for split classical groups in [Mou, §4.2.3].

The desiderata of the Langlands correspondence show how "inertial equivalence" can be be formulated for L-parameters. Let $\mathbf{I}_{F}$ be the inertia subgroup of $\mathbf{W}_{F}$ and 
let Frob $_{F} \in \mathbf{W}_{F}$ be a Frobenius element, so that

$$
\mathbf{W}_{F} / \mathbf{I}_{F} \cong\left\langle\operatorname{Frob}_{F}\right\rangle \cong \mathbb{Z} \text {. }
$$

By [Hai, (3.3.2)] there are natural isomorphisms

$$
\left.X_{\mathrm{nr}}(G) \cong\left(Z\left(G^{\vee}\right)^{\mathbf{I}_{F}}\right)_{\left\langle\operatorname{Frob}_{F}\right\rangle}^{\circ} \cong H_{c}^{1}\left(\mathbf{W}_{F} / \mathbf{I}_{F}, Z\left(G^{\vee}\right)^{\mathbf{I}_{F}}\right)^{\circ}\right) .
$$

We will denote a typical cuspidal L-parameter by $(\varphi, \varepsilon) \in \Phi_{\text {cusp }}(L) \subset \Phi_{e}(L)$. In view of Borel's desideratum (2) for Conjecture 1, [Hai, 5.3.3] and [Mou, Def. 4.15], we define $(L, \phi, \varepsilon),\left(L^{\prime}, \varphi^{\prime}, \varepsilon^{\prime}\right)$ to be inertially equivalent (for $G^{\vee}$ ) if there exist $g \in G^{\vee}$ and $z \in H_{c}^{1}\left(\mathbf{W}_{F} / \mathbf{I}_{F},\left(Z\left(L^{\vee}\right)^{\mathbf{I}_{F}}\right)^{\circ}\right)$ such that

$$
L^{\prime \vee}={ }^{g} L^{\vee}, \quad \varphi^{\prime}=z^{g} \varphi, \quad \varepsilon^{\prime}={ }^{g} \varepsilon .
$$

We denote their inertial equivalence class by $\mathfrak{s}^{\vee}=\left[L^{\vee}, \varphi, \varepsilon\right]_{G^{\vee}}$, and we let $\mathfrak{B}^{\vee}(G)$ be the collection of inertial equivalence classes.

The analogue of a Bernstein component in $\Phi_{e}(G)$ should be

$$
\Phi_{e}(G)^{\mathfrak{s}^{\vee}}=\left\{(\phi, \rho) \in \Phi_{e}(G) \text { : the cuspidal support of }(\phi, \rho) \text { lies in } \mathfrak{s}^{\vee}\right\} .
$$

Of course this is only meaningful if the cuspidal support of enhanced Langlands parameters can be defined precisely. We expect that under the $\operatorname{LLC} \Phi_{e, \zeta_{G}}(G)^{\mathfrak{s}^{\vee}}$ will be in bijection with $\operatorname{Irr}(G)^{\mathfrak{s}}$, where $\mathfrak{s}=[L, \omega]_{G}$ with $\omega \in \operatorname{Irr}_{\text {cusp }}(L)$ corresponding to some $(\varphi, \varepsilon) \in \mathfrak{s}^{\vee}$.

One may wonder how $W(G, L)$ acts on $\Phi_{\text {cusp }}(L)$ in (38). That should come from the action of $N_{G^{\vee}}\left(L^{\vee} \rtimes \mathbf{W}_{F}\right)$ on $\Phi_{e}(L)$, via the next result.

Proposition 3.1. Let $L$ be any Levi subgroup of $G$. There is a canonical isomorphism

$$
W(G, L) \cong N_{G^{\vee}}\left(L^{\vee} \rtimes \mathbf{W}_{F}\right) / L^{\vee} .
$$

Proof. First we reformulate $W(G, L)$ in terms of the root datum of $\mathcal{G}$. Let $S=\mathcal{S}(F)$ be a maximal $F$-split torus in $L=\mathcal{L}(F)$. The relative (with respect to $F$ ) Weyl group of $G=\mathcal{G}(F)$ is

$$
W(G, S)=N_{G}(S) / Z_{G}(S)
$$

Both the canonical maps

$$
\left(\operatorname{Stab}_{N_{G}(S)}(L) / Z_{G}(S)\right) /\left(N_{L}(S) / Z_{L}(S)\right) \rightarrow \operatorname{Stab}_{N_{G}(S)}(L) / N_{L}(S) \rightarrow N_{G}(L) / L
$$

are bijective, the last one because all maximal $F$-split tori in $L$ are $L$-conjugate [Spr, Theorem 15.2.6]. In other words,

$$
\operatorname{Stab}_{W(G, S)}(L) / W(L, S) \cong W(G, L) .
$$

Let $\mathcal{T}$ be a maximal $F$-torus of $\mathcal{L}$ containing $\mathcal{S}$. The absolute Weyl group $W(\mathcal{G}, \mathcal{T})=$ $N_{\mathcal{G}}(\mathcal{T}) / \mathcal{T}$ is endowed with an action of $\mathbf{W}_{F}$. The relative Weyl group is the restriction of $W(\mathcal{G}, \mathcal{T})^{\mathbf{W}_{F}}$ to $X^{*}(\mathcal{S})$ [Spr, $\left.\S 15.3\right]$. That is,

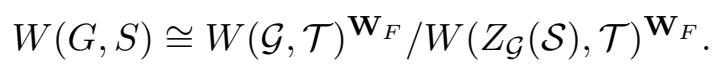

An element of $N_{\mathcal{G}}(\mathcal{T})$ normalizes $\mathcal{L}$ if and only if it stabilizes the root subsytem $R(\mathcal{L}, \mathcal{T}) \subset R(\mathcal{G}, \mathcal{T})$. Combining (41) and (42), we find

$$
W(G, L) \cong \operatorname{Stab}_{W(\mathcal{G}, \mathcal{T})^{\mathbf{w}_{F}}}(R(\mathcal{L}, \mathcal{T})) / W(\mathcal{L}, \mathcal{T})^{\mathbf{W}_{F}}
$$


Now we are in a good position to pass to the complex dual groups. Using the canonical isomorphism

$$
W(\mathcal{G}, \mathcal{T}) \cong W\left(\mathcal{G}^{\vee}, \mathcal{T}^{\vee}\right)=W\left(G^{\vee}, T^{\vee}\right),
$$

we obtain

$$
W(G, L) \cong \operatorname{Stab}_{W\left(G^{\vee}, T^{\vee}\right)} \mathbf{w}_{F}\left(R\left(L^{\vee}, T^{\vee}\right)\right) / W\left(L^{\vee}, T^{\vee}\right)^{\mathbf{w}_{F}} .
$$

Because $\mathcal{T}$ is defined over $F, T^{\vee}$ is $\mathbf{W}_{F}$-stable and we can form $T^{\vee} \rtimes \mathbf{W}_{F}$. An element of $N_{G^{\vee}}\left(T^{\vee}\right)$ is fixed by $\mathbf{W}_{F}$ if and only if it normalizes $T^{\vee} \rtimes \mathbf{W}_{F}$.

Inside the Langlands dual group $G^{\vee} \rtimes \mathbf{W}_{F}$ we can rewrite the right hand side of (43) as

$$
\begin{aligned}
\left(\operatorname{Stab}_{N_{G} \vee}\left(T^{\vee} \mathbf{W}_{F}\right)\right. & \left.\left(R\left(L^{\vee}, T^{\vee}\right)\right) / T^{\vee}\right) /\left(N_{L^{\vee}}\left(T^{\vee} \rtimes \mathbf{W}_{F}\right) / T^{\vee}\right) \\
& \cong \operatorname{Stab}_{N_{G} \vee}\left(T^{\vee} \rtimes \mathbf{W}_{F}\right) \\
& \left(R\left(L^{\vee}, T^{\vee}\right)\right) /\left(N_{L^{\vee}}\left(T^{\vee} \rtimes \mathbf{W}_{F}\right) .\right.
\end{aligned}
$$

A standard argument shows that the canonical injection

$$
\operatorname{Stab}_{N_{G} \vee}\left(T^{\vee} \rtimes \mathbf{W}_{F}\right)\left(R\left(L^{\vee}, T^{\vee}\right)\right) /\left(N_{L^{\vee}}\left(T^{\vee} \rtimes \mathbf{W}_{F}\right)\right) \rightarrow N_{G^{\vee}}\left(L^{\vee} \rtimes \mathbf{W}_{F}\right) / L^{\vee}
$$

is surjective. Namely, for $n \in N_{G^{\vee}}\left(L^{\vee} \rtimes \mathbf{W}_{F}\right), n T^{\vee} n^{-1}$ is a maximal torus of the complex group $L^{\vee}$, so it is conjugate to $T^{\vee}$ by some $l \in L^{\vee}$. Then

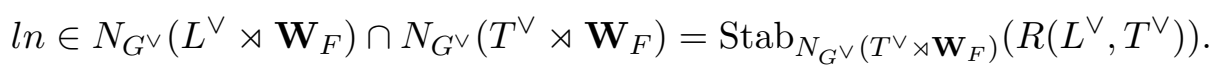

Hence $W(G, L)$ is canonically isomorphic to the right hand sides of (44) and (45).

Now we have a well-defined action of $W(G, L) \cong N_{G^{\vee}}\left(L^{\vee} \rtimes \mathbf{W}_{F}\right) / L^{\vee}$ on

$$
\Phi_{\text {cusp }}(L)=\bigsqcup_{\mathfrak{s}_{L}^{\vee}=\left[L^{\vee}, \varphi, \varepsilon\right]_{L^{\vee}}} \Phi_{e}(L)^{\mathfrak{s}^{\vee}} \text {. }
$$

The action preserves this decomposition because it stabilizes the group of unramified characters $X_{\mathrm{nr}}(L) \cong H_{c}^{1}\left(\mathbf{W}_{F} / \mathbf{I}_{F},\left(Z\left(L^{\vee}\right)^{\mathbf{I}_{F}}\right)^{\circ}\right)$. Hence we can transfer the definition of Bernstein's finite group $W_{\mathfrak{s}}$ to the Galois side. For $\mathfrak{s}^{\vee}=\left[L^{\vee}, \varphi, \varepsilon\right]_{G^{\vee}} \in \mathfrak{B}^{\vee}(G)$ we define:

$$
W_{\mathfrak{s}^{\vee}}=\text { stabilizer of } \Phi_{e}(L)^{\mathfrak{s} L} \text { in } N_{G^{\vee}}\left(L^{\vee} \rtimes \mathbf{W}_{F}\right) / L^{\vee} .
$$

It is expected (and proved in [Mou, Théorème 5.6] in the case of split groups of classical type) that if $\sigma \in \operatorname{Irr}(L)$ corresponds to $(\varphi, \varepsilon) \in \Phi_{\text {cusp }}(L)$ via LLC then the groups $W_{\mathfrak{s}}$ and $W_{\mathfrak{s} \vee}$ are isomorphic.

\subsection{Langlands parameters and extended quotients.}

It is reasonable to expect that the conjectural bijection

$$
\Phi_{e}(G)^{\mathfrak{s}} \longleftrightarrow\left(\Phi_{e}(L)^{\mathfrak{s} L} / / W_{\mathfrak{s}}\right)_{\mathfrak{\natural}}
$$

from (37) can be constructed purely in terms of Langlands parameters, without using $p$-adic groups. Indeed, this was already done for $\mathrm{GL}_{n}(F)$ in $[\mathrm{BrPl}, \S 1]$. Let us give two more examples.

Example. Let $G=\mathrm{SL}_{2}(F), G^{\vee}=\mathrm{PGL}_{2}(\mathbb{C})$ and $L=T \cong F^{\times}$. We record that $W\left(G^{\vee}, T^{\vee}\right) \cong W_{\mathfrak{s}} \cong \mathbb{Z} / 2 \mathbb{Z}$, where $\operatorname{Irr}^{\mathfrak{s}}(G)$ is Iwahori-spherical Bernstein component. For $\phi$ we simply take the unit map $\mathbf{W}_{F} \times \mathrm{SL}_{2}(\mathbb{C}) \rightarrow T^{\vee} \subset G^{\vee}$. Then $\phi \in \Phi_{\text {cusp }}(T)$ and $\left(\phi, \operatorname{sign}_{W_{\mathfrak{s}}}\right) \in\left(\Phi_{e}(T)^{\mathfrak{s} T} / / W_{\mathfrak{s}}\right)_{2}$. 
From this we want to construct $(\tilde{\phi}, \rho) \in \Phi_{e}(G)^{\mathfrak{s}}$. The Springer correspondence for $W_{\mathfrak{s}}$ associates to the sign representation the conjugacy class of the unipotent element $u=\left(\begin{array}{ll}1 & 1 \\ 0 & 1\end{array}\right) \in \mathrm{PGL}_{2}(\mathbb{C})$. We define $\tilde{\phi}$ by

$$
\left.\tilde{\phi}\right|_{\mathbf{W}_{F}}=\left.\phi\right|_{\mathbf{W}_{F}}=1 \text { and } \tilde{\phi}\left(1,\left(\begin{array}{ll}
1 & 1 \\
0 & 1
\end{array}\right)\right)=u \text {. }
$$

For a lack of choice we have to take $\rho=1$. Notice that this agrees with the example on page 19 and with the LLC for $\mathrm{SL}_{2}(F)$ : both $\left(\phi, \operatorname{sign}_{W_{\mathfrak{s}}}\right)$ and $\tilde{\phi}$ correspond to the Steinberg representation.

Example. Let $G=\mathrm{GL}_{m}(D)$ and let $\chi \in \operatorname{Irr}\left(\mathrm{SL}_{m d}(\mathbb{C})\right)$ be the character that defines $G$ as an inner twist of $\operatorname{GL}_{m d}(F)$ (see page 6). Assume that $\phi$ is a Langlands parameter for a supercuspidal representation of a standard Levi subgroup $L=\prod_{i} \mathrm{GL}_{m_{i}}(F)^{e_{i}}$ of $G$, of the form $\prod_{i} \phi^{e_{i}}$ with $\phi_{i}: \mathbf{W}_{F} \times \mathrm{SL}_{2}(\mathbb{C}) \rightarrow \mathrm{GL}_{m_{i} d}(\mathbb{C})$ discrete.

Since $\mathfrak{R}_{\phi}=1$ for all $\phi \in \Phi\left(\mathrm{GL}_{m d}(F)\right)$, and by Lemma 1.5 , we have $\mathcal{Z}_{\phi}=\mathcal{S}_{\phi}$ and $(\phi, \chi) \in \Phi_{e}(L)^{\mathfrak{s}^{\vee}}$. The stabilizer of $\phi$ in $W_{\mathfrak{s}^{\vee}}$ is $W_{\mathfrak{s}^{\vee}, \phi} \cong \prod_{i} S_{e_{i}}$. Let $\rho \in \operatorname{Irr}\left(W_{\mathfrak{s}^{\vee}, \phi}\right)$, so that $(\phi, \chi, \rho) \in\left(\Phi_{e}(L)^{\mathfrak{s} L} / / W_{\mathfrak{s}}\right)_{2}$.

To construct an element of $\Phi_{e}(G)^{\mathfrak{s}^{\vee}}$ from this we proceed as above, only with more data. Via the Springer correspondence for $W_{\mathfrak{s}, \phi}, \rho$ determines a unipotent class $[u]$ in $Z_{\mathrm{GL}_{m d}(\mathbb{C})}(\phi) \cong \prod_{i} \mathrm{GL}_{e_{i}}(\mathbb{C})$. We put

$$
\left.\tilde{\phi}\right|_{\mathbf{W}_{F}}=\left.\phi\right|_{\mathbf{W}_{F}} \text { and } \tilde{\phi}\left(1,\left(\begin{array}{ll}
1 & 1 \\
0 & 1
\end{array}\right)\right)=u \phi\left(1,\left(\begin{array}{ll}
1 & 1 \\
0 & 1
\end{array}\right)\right) \text {. }
$$

Then $(\tilde{\phi}, \chi) \in \Phi_{e}(G)^{\mathfrak{s}^{\vee}}$ and with $[\mathrm{ABPS7}$, Theorem 5.3] one can check that it corresponds to the same $G$-representation as $(\phi, \chi, \rho)$.

With all the notions from the previous paragraph we can formulate a Galois version of Conjecture 2, see [Mou, §5.3].

Conjecture 4. Let $L$ be any Levi subgroup of $G$ and let $\mathfrak{s}^{\vee}=\left[L^{\vee}, \varphi, \varepsilon\right]_{G^{\vee}} \in \mathfrak{B}^{\vee}(G)$. There exists a family of 2-cocycles $\square$ and bijections

$$
\begin{aligned}
\Phi_{e, \zeta_{G}}(G)^{\mathfrak{s}^{\vee}} & \longleftrightarrow\left(\Phi_{e, \zeta_{G}}(L)^{\mathfrak{s}^{\vee}} / / W_{\mathfrak{s} \vee}\right)_{\mathfrak{\natural}}, \\
\bigsqcup_{\mathfrak{S}^{\vee}=\left[L^{\vee}, \varphi, \varepsilon\right]_{G} \vee} \Phi_{e, \zeta_{G}}(G)^{\mathfrak{s}^{\vee}} & \longleftrightarrow\left(\Phi_{\operatorname{cusp}, \zeta_{G}}(L) / / W(G, L)\right)_{\mathfrak{\natural}} .
\end{aligned}
$$

Moreover these maps preserve boundedness, and they can be constructed entirely in terms of complex reductive groups with $\mathbf{W}_{F}$-actions.

This conjecture was proven for split classical groups in [Mou, Théorème 5.5] and for principal series Bernstein components of split reductive groups in [ABPS6, 55 and Theorem 8.2].

We note that the two bijections in Conjecture 4 are the same, since by the definition of $W_{\mathfrak{s} \vee}$ the canonical map

$$
\bigsqcup_{\mathfrak{s} \vee=\left[L^{\vee}, \varphi, \varepsilon\right]_{G} \vee}\left(\Phi_{e, \zeta_{G}}(L)^{\mathfrak{s}^{\vee}} / / W_{\mathfrak{s} \vee}\right)_{\natural} \longrightarrow\left(\Phi_{\operatorname{cusp}, \zeta_{G}}(L) / / W(G, L)\right)_{\natural}
$$

is a bijection. It seems that Conjecture 4 uses the $p$-adic groups $G$ and $L$, but this is only notational. All the relevant objects are defined in terms of ${ }^{L} G$, the character $\zeta_{G}$ of $Z\left(G_{\mathrm{sc}}^{\vee}\right)$, and the Levi subgroup $L^{\vee} \rtimes \mathbf{W}_{F} \subset{ }^{L} G$. 


\section{TOPOLOGical K-THEORY}

We discuss the K-theory of the reduced $C^{*}$-algebra of $G$. Different pictures of these groups are provided by several conjectures: the Baum-Connes conjecture, Conjecture 2 and the local Langlands correspondence (although only in an heuristic way).

\subsection{Equivariant K-theory.}

This paragraph is a counterpart to paragraph 2.1. We work in the same generality, just with groups acting on nice spaces, and we end up with the topological K-theory of extended quotients.

Let $X$ be a locally compact Hausdorff space and let $\Gamma$ be a group acting on $X$. For simplicity we assume that $\Gamma$ is finite. The $\Gamma$-equivariant $\mathrm{K}$-theory of $X$ was defined in [Ati, §2.4]. When $X$ is compact, $K_{\Gamma}^{0}(X)$ is the Grothendieck group of the semigroup of complex $\Gamma$-vector bundles on $X$. When $X$ is only locally compact, we let $X \cup\{\infty\}$ be its one-point compactification, and we put

$$
K_{\Gamma}^{0}(X)=\operatorname{ker}\left(K_{\Gamma}^{0}(X \cup\{\infty\}) \rightarrow K_{\Gamma}^{0}(\{\infty\})\right) .
$$

The equivariant $K^{1}$-group is defined via the suspension functor. It can be expressed as

$$
K_{\Gamma}^{1}(X)=K_{\Gamma}^{0}(X \times \mathbb{R})
$$

where $\Gamma$ acts trivially on $\mathbb{R}$. Typically one writes

$$
K_{\Gamma}^{*}(X)=K_{\Gamma}^{0}(X) \oplus K_{\Gamma}^{1}(X),
$$

a $\mathbb{Z} / 2 \mathbb{Z}$-graded abelian group. Let

$$
C_{0}(X)=\{f \in C(X \cup\{\infty\}, \mathbb{C}): f(\infty)=0\}
$$

be the commutative $C^{*}$-algebra of functions on $X$ which vanish at infinity. By the Serre-Swan Theorem its K-theory is

$$
K_{*}\left(C_{0}(X)\right) \cong K^{*}(X) .
$$

The group $\Gamma$ acts on $C_{0}(X)$ by automorphisms, and we form the crossed product $C_{0}(X) \rtimes \Gamma$. Recall from Lemma 2.1 that $\operatorname{Irr}\left(C_{0}(X) \rtimes \Gamma\right) \cong(X / / \Gamma)_{2}$. By the GreenJulg Theorem [Jul] and the equivariant Serre-Swan Theorem [Phi, 2.3.1] there is a natural isomorphism

$$
K_{*}\left(C_{0}(X) \rtimes \Gamma\right) \cong K_{\Gamma}^{*}(X) .
$$

Thus we can interpret $K_{\Gamma}^{*}(X)$ as the K-theory of the topological space $(X / / \Gamma)_{2}$. Of course that space is usually not Hausdorff, so the statement is not precise, it is rather a manifestation of the philosophy of noncommutative geometry.

Now we consider twisted extended quotients. Let $\downarrow: \Gamma \times \Gamma \rightarrow \mathbb{C}^{\times}$be a 2-cocycle. As in (23), we can find a central extension

$$
1 \rightarrow Z \rightarrow \tilde{\Gamma} \rightarrow \Gamma \rightarrow 1
$$

a character $\chi_{\natural}$ of $Z$ and a minimal idempotent $p_{\natural} \in \mathbb{C}[Z]$ such that $p_{\natural} \mathbb{C}[\tilde{\Gamma}] \cong \mathbb{C}[\Gamma, \emptyset]$.

The group $\tilde{\Gamma}$ also acts on $X$, via its projection to $\Gamma$. Then $C_{0}(X) \rtimes p_{\natural} \mathbb{C}[\tilde{\Gamma}]$ is a direct summand of $C_{0}(X) \rtimes \tilde{\Gamma}=C_{0}(X) \rtimes \mathbb{C}[\tilde{\Gamma}]$. It follows from (47) that

$$
K_{*}\left(C_{0}(X) \rtimes p_{\natural} \mathbb{C}[\tilde{\Gamma}]\right) \cong p_{\natural} K_{\tilde{\Gamma}}^{*}(X) .
$$


In view of Lemma 2.3 , the left hand side can be regarded as the K-theory of the topological space $(X / / \Gamma)_{\mathfrak{t}}$. The right hand side of (49) also admits a geometric interpretation. We saw in $(46)$ that $K_{\tilde{\Gamma}}^{0}(X)$ is built from $\tilde{\Gamma}$-vector bundles on $X$. The central idempotent $p_{\natural}$ selects the direct summands corresponding to the $\tilde{\Gamma}$ vector bundles on which $Z$ acts as $\chi_{\natural}$. Similarly, $K_{\Gamma}^{1}(X)$ can be constructed from the semigroup of $\tilde{\Gamma}$-vector bundles on $X \rtimes \mathbb{R}$ on which $Z$ acts as $\chi_{\natural}$. These semigroups of vector bundles depend on $X, \Gamma$ and $\boxminus$, but not on the central extension $\tilde{\Gamma}$ chosen to analyse $\downarrow$. Thus we can define the $\downarrow$-twisted $\Gamma$-equivariant $K$-theory of $X$ as

$$
K_{\Gamma, \natural}^{*}(X):=p_{\natural} K_{\tilde{\Gamma}}^{*}(X) .
$$

Then, loosely speaking,

$$
K^{*}\left((X / / \Gamma)_{\natural}\right) \cong K_{\Gamma, \natural}^{*}(X)
$$

\subsection{The Baum-Connes conjecture.}

As before, let $G=\mathcal{G}(F)$ be a reductive $p$-adic group. The reduced $C^{*}$-algebra $C_{r}^{*}(G)$ is the completion of $\mathcal{H}(G)$ in the algebra of bounded linear operators on the Hilbert space $L^{2}(G)$. It follows from the work of Harish-Chandra (see [Vig, $\S 10])$ that the irreducible representations of $C_{r}^{*}(G)$ can be identified with those of the Schwartz algebra of $G$. By [Wal, §III.7] the latter are the same as irreducible tempered $G$-representations. Thus we get

$$
\operatorname{Irr}\left(C_{r}^{*}(G)\right)=\operatorname{Irr}_{\text {temp }}(G)
$$

which means that $C_{r}^{*}(G)$ is the correct $C^{*}$-algebra to study the noncommutative geometry of the tempered dual of $G$. The structure of $C_{r}^{*}(G)$ was described by means of the Fourier transform in [Ply].

The Baum-Connes conjecture provides a picture of the $K$-theory of this $C^{*}$ algebra in geometric terms. Let $\mathcal{B}(G)$ be the (nonreduced) affine building of $G$, as developed by Bruhat and Tits [BrTi1, BrTi2]. This is a proper $G$-space with many remarkable properties, for example:

- $\mathcal{B}(G)$ satisfies the negative curvature inequality [Tit, 2.3] and hence is contractible and has unique geodesics [Bro, §VI.3];

- every compact subgroup of $G$ fixes a point of $\mathcal{B}(G)$, see [Tit, §2.3.1] or [Bro, $\S$ VI.4].

In view of [BCH, Proposition 1.8], these properties make $\mathcal{B}(G)$ into a universal space for proper $G$-actions [BCH, Definition 1.6].

The $G$-equivariant K-homology $K_{*}^{G}(\mathcal{B}(G))$ of the building was defined in [BCH, $\S 3]$. The Baum-Connes conjecture asserts that the canonical assembly map

$$
K_{*}^{G}(\mathcal{B}(G)) \rightarrow K_{*}\left(C_{r}^{*}(G)\right)
$$

is an isomorphism. This was proven (for a large class of groups containing $G$ ) in [Laf1]. For the groups under consideration the Baum-Connes conjecture can also be formulated and proven more algebraically [HiNi, Schn], with equivariant cosheaf homology (also known as chamber homology) [ABP, §2]. By [Sol1] these two versions of the conjecture are compatible.

The left-hand-side of (52), defined in terms of $K$-cycles, has never been directly computed for a noncommutative reductive $p$-adic group. Results of Voigt [Voi] allow us to replace the left-hand-side with the chamber homology groups. Chamber homology has been directly computed for only two noncommutative $p$-adic groups: 
$\mathrm{SL}_{2}(F)$ [BHP1] and $\mathrm{GL}_{3}(F)[\mathrm{AHP}]$. In the case of $\mathrm{GL}_{3}(F)$, one can be sure that representative cycles in all the homology groups have been constructed only by checking with the right-hand-side of the Baum-Connes conjecture. In other words, one always has to have an independent computation of the right-hand-side.

On the $C^{*}$-algebra of (52) side our earlier conjectures have something to say. The Bernstein decomposition of $\mathcal{H}(G)(33)$ gives rise to a factorization

$$
C_{r}^{*}(G)=\prod_{\mathfrak{s} \in \mathfrak{B}(G)} C_{r}^{*}(G)^{\mathfrak{s}} \quad \text { with } \quad \operatorname{Irr}\left(C_{r}^{*}(G)^{\mathfrak{s}}\right)=\operatorname{Irr}_{\text {temp }}(G)^{\mathfrak{s}}
$$

Morally speaking, $K_{*}\left(C_{r}^{*}(G)^{\mathfrak{s}}\right)$ is the K-theory of the topological space $\operatorname{Irr}_{\text {temp }}(G)^{\mathfrak{s}}$. Combining this with Conjecture 2 and (50) leads to:

Conjecture 5. Let $\mathfrak{s} \in \mathfrak{B}(G)$. There exists a canonical isomorphism

$$
K_{W_{\mathfrak{s}}, \mathfrak{\natural}}^{*}\left(T_{\mathfrak{s}, \text { un }}\right) \rightarrow K_{*}\left(C_{r}^{*}(G)^{\mathfrak{s}}\right) .
$$

This is the topological K-theory version of Conjecture 2. Of course it is much weaker, since it only says something about the cohomology of $\left(T_{\mathfrak{s}} / / W_{\mathfrak{s}}\right)_{\mathfrak{t}}$, and not so much about the space itself. Yet in practice, with some additional knowledge of the underlying algebras, this already provides a lot of information. Conjecture 5 provides a much finer and more precise formula for $K_{*}\left(C_{r}^{*}(G)\right)$ than Baum-Connes alone.

Let us consider the reduced Iwahori-spherical $C^{*}$-algebra $C_{r}^{*}(G)^{\mathfrak{i}} \subset C_{r}^{*}(G)$ in more detail. The primitive ideal spectrum of $C_{r}^{*}(G)^{\mathfrak{i}}$ can be identified with the irreducible tempered representations of $G$ which admit nonzero Iwahori-fixed vectors. We assume that $G$ is split, so $\mathfrak{i}=[T, 1]_{G}$ and $T_{\mathfrak{i}}=T^{\vee}$ is a maximal torus in the complex dual group $G^{\vee}$. In this special case, Conjecture 5 asserts that

$$
K_{j}\left(C_{r}^{*}(G)^{\mathfrak{i}}\right) \cong K_{W_{\mathfrak{i}}}^{j}\left(T_{u n}^{\vee}\right)
$$

with $j=0,1$. Here $K_{W_{\mathrm{i}}}^{j}\left(T_{u n}^{\vee}\right)$ is the classical topological equivariant K-theory for the Weyl group $W_{\mathfrak{i}} \cong W\left(G^{\vee}, T^{\vee}\right)$ acting on the compact torus $T_{u n}^{\vee}=T_{\mathfrak{i}, u n}$.

Let $X^{*}\left(T_{u n}^{\vee}\right)$ denote the group of Lie group morphisms from $X^{*}\left(T_{u n}^{\vee}\right)$ to $\mathrm{U}(1)$, that is, $X^{*}\left(T_{u n}^{\vee}\right)$ denotes the Pontryagin dual of $T_{u n}^{\vee}$. It is naturally isomorphic with the lattice of algebraic characters of $T^{\vee}$. We have

$$
C_{r}^{*}\left(X^{*}\left(T_{u n}^{\vee}\right) \rtimes W_{\mathfrak{i}}\right) \cong C\left(T_{u n}^{\vee}\right) \rtimes W_{\mathfrak{i}}
$$

by a standard Fourier transform. By (35) and [Sol2, Theorem 5.1.4]

$$
K_{j}\left(C_{r}^{*}(G)^{\mathfrak{i}}\right) \otimes_{\mathbb{Z}} \mathbb{Q} \cong K_{j}\left(C_{r}^{*}\left(X^{*}\left(T_{u n}^{\vee}\right) \rtimes W_{\mathfrak{i}}\right)\right) \otimes_{\mathbb{Z}} \mathbb{Q},
$$

where $j=0,1$. With (47) we get

$$
K_{j}\left(C_{r}^{*}(G)^{\mathfrak{i}}\right) \otimes_{\mathbb{Z}} \mathbb{Q} \cong K_{W_{\mathfrak{i}}}^{j}\left(T_{u n}^{\vee}\right) \otimes_{\mathbb{Z}} \mathbb{Q},
$$

which establishes (53) modulo torsion.

In general, if Conjecture 3 would hold for $\mathfrak{s}$, then $C_{r}^{*}(G)^{\mathfrak{s}}$ would be Morita equivalent with $e_{\mathfrak{s}} C_{r}^{*}(G) e_{\mathfrak{s}}$, and that algebra could be described in terms of $C^{*}$-completions of affine Hecke algebras [Opd]. With the techniques developed in [Sol1, §5.1] and $[$ ABPS7, $\S 6]$ that would go a long way towards Conjecture 5 .

Now two pictures of $K_{*}\left(C_{r}^{*}(G)\right)$ are available, namely $K_{*}^{G}(\mathcal{B}(G))$ and $\bigoplus_{\mathfrak{s} \in \mathfrak{B}(G)} K_{W_{\mathfrak{s}, \mathfrak{h}}}^{*}\left(T_{\mathfrak{s}, \text { un }}\right)$. Unfortunately they are not compatible in any obvious way. 
It is even unclear how a Bernstein decomposition of $K_{*}^{G}(\mathcal{B}(G))$ would look like, see $[\mathrm{BHP} 2, \S 5]$ for a discussion of the analogous problem in chamber homology.

We sketch how some comparisons can be made. Let $S$ be a maximal $F$-split torus of $G$ and let $A_{S}=X_{*}(S) \otimes_{\mathbb{Z}} \mathbb{R}$ be the corresponding apartment of $\mathcal{B}(G)$. It is endowed with an action of

$$
W^{e}(G, S):=N_{G}(S) / Z_{G}(S)_{\mathrm{cpt}} \cong Z_{G}(S) / Z_{G}(S)_{\mathrm{cpt}} \rtimes W(G, S),
$$

a group which contains $X_{*}(S) \rtimes W(G, S)$ as a subgroup of finite index. More generally, for any Levi subgroup $L \subset G$ the group $W^{e}(L, S)$ acts on $A_{S}$, and $A_{S}$ is a universal example for proper $W^{e}(L, S)$-actions.

Let $\mathfrak{s}=[L, \omega]_{G}$ and regard $\omega$ as the basepoint of $T_{\mathfrak{s}, \text { un }}$. Via $(24)$ this turns $T_{\mathfrak{s}, \text { un }}$ into a Lie group, so we can speak of its characters. Then

$$
K_{W_{\mathfrak{s}}, \mathfrak{t}}^{*}\left(T_{\mathfrak{s}, \text { un }}\right) \cong K_{*}\left(C\left(T_{\mathfrak{s}, \text { un }}\right) \rtimes \mathbb{C}\left[W_{\mathfrak{s}}, \mathfrak{\natural}\right]\right) \cong K_{*}\left(C_{r}^{*}\left(X^{*}\left(T_{\mathfrak{s}, \text { un }}\right)\right) \rtimes \mathbb{C}\left[W_{\mathfrak{s}}, \mathfrak{\natural}\right]\right) .
$$

Choosing a central extension as in (48), we can rewrite the right hand side of (55) as

$$
K_{*}\left(p_{\natural} C_{r}^{*}\left(X^{*}\left(T_{\mathfrak{s}, \text { un }}\right) \rtimes \tilde{W}_{\mathfrak{s}}\right)\right)=p_{\natural} K_{*}\left(C_{r}^{*}\left(X^{*}\left(T_{\mathfrak{s}, \text { un }}\right) \rtimes \tilde{W}_{\mathfrak{s}}\right)\right) .
$$

It follows from [Ren, $\S \mathrm{V} .2 .6]$ that the character lattice $X^{*}\left(T_{\mathfrak{s}, \text { un }}\right)=X^{*}\left(T_{\mathfrak{s}}\right)$ is naturally isomorphic to a cocompact subgroup of

$$
A_{Z(L)}=\left(Z(L) / Z(L)_{\mathrm{cpt}}\right) \otimes_{\mathbb{Z}} \mathbb{R},
$$

So the Baum-Connes conjecture for $X^{*}\left(T_{\mathfrak{s}, \text { un }}\right) \rtimes \tilde{W}_{\mathfrak{s}}$ gives isomorphisms

$$
K_{W_{\mathfrak{s}}, \mathfrak{}}^{*}\left(T_{\mathfrak{s}, u n}\right) \cong p_{\natural} K_{*}\left(C_{r}^{*}\left(X^{*}\left(T_{\mathfrak{s}, u n}\right) \rtimes \tilde{W}_{\mathfrak{s}}\right)\right) \cong p_{\natural} K_{*}^{X^{*}\left(T_{\mathfrak{s}, u n}\right) \rtimes \tilde{W}_{\mathfrak{s}}}\left(A_{Z(L)}\right) .
$$

The canonical embedding $A_{Z(L)} \rightarrow A_{S} \subset \mathcal{B}(G)$ should identify the isotropy groups for the action of $X^{*}\left(T_{\mathfrak{s}, \text { un }}\right) \rtimes W_{\mathfrak{s}}$ acting on $A_{Z(L)}$ with subquotients of the isotropy groups of $G$ acting on $\mathcal{B}(G)$. Via (56) that should give a map

$$
K_{W_{\mathfrak{s}, \mathfrak{l}}}^{*}\left(T_{\mathfrak{s}, \text { un }}\right) \rightarrow K_{*}^{G}(\mathcal{B}(G)) .
$$

Of course this construction is too simple, because it does not take the $L$-representation $\omega$ into account. Yet at least it gives us a geometric idea of how the two pictures of $K_{*}\left(C_{r}^{*}(G)\right)$ can be related. Probably a good map from $K_{*}\left(C_{r}^{*}(G)^{\mathfrak{s}}\right)$ to $K_{*}^{G}(\mathcal{B}(G))$ will have to involve a nice idempotent for $\mathfrak{s}$. But, even with a $\mathfrak{s}$-type available the issue is currently unclear.

\subsection{Relations with the LLC.}

Recall that Conjecture 1 predicts a bijection

$$
\operatorname{Irr}_{\text {temp }}(G) \longleftrightarrow \Phi_{e, \zeta_{G}, \text { bdd }}(G),
$$

where the subscript "bdd" indicates bounded L-parameters. By $(51) K_{*}\left(C_{r}^{*}(G)\right)$ can be regarded as the K-theory of $\operatorname{Irr}_{\text {temp }}(G)$. It is not so clear what the (topological) $\mathrm{K}$-theory of $\Phi_{e, \zeta_{G} \text {,bdd }}(G)$ should be, because there is no convenient algebra in sight. Here Conjecture 4 is useful. As it respects boundedness of L-parameters, it predicts a bijection

$$
\Phi_{e, \zeta_{G}, \text { bdd }}(G) \longleftrightarrow \bigsqcup_{\mathfrak{s}^{\vee}=\left[L^{\vee}, \varphi, \varepsilon\right]_{G} \vee \in \mathfrak{B}^{\vee}(G)}\left(\Phi_{e, \zeta_{G}, \operatorname{bdd}}(L)^{\mathfrak{s}^{\vee}} / / W_{\mathfrak{s}^{\vee}}\right)_{\mathfrak{\natural}} .
$$


The K-theory of the right hand side can be interpreted with (50). The LLC for $\operatorname{Irr}_{\text {cusp }}(L)$ should provide a $W(G, L)$-equivariant bijection

$$
\operatorname{Irr}_{\text {cusp }, \text { temp }}(L) \longleftrightarrow \Phi_{\text {cusp }, \zeta_{G}, \text { bdd }}(L),
$$

which induces a group isomorphism $W_{\mathfrak{s}} \cong W_{\mathfrak{s} \vee}$ if $T_{\mathfrak{s}}$ corresponds to $\Phi_{e, \zeta_{G}}(L)^{\mathfrak{s} \frac{\mathfrak{s}}{L}}$.

Let us combine all these descriptions of $K_{*}\left(C_{r}^{*}(G)\right)$ in one diagram:

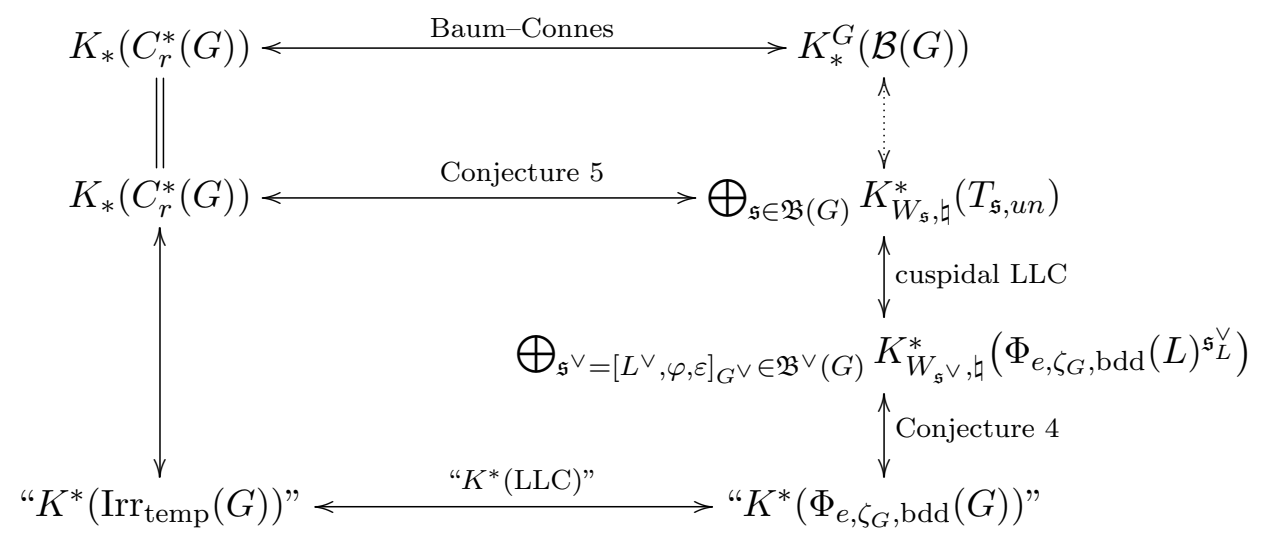

On the top of the right hand side we have the " $p$-adic" geometry of the BruhatTits building of $G$, combined with the noncommutative geometry from equivariant K-homology. At the bottom we find, in some sense, the cohomology of the space of enhanced bounded L-parameters for $G$. The extended quotients obtained from the Bernstein decomposition for $G$ interpolate between these very different settings. In this way our Conjectures 4 and 5 connect the Baum-Connes conjecture and the local Langlands correspondence.

\section{REFERENCES}

[Art1] J. Arthur, "On the transfer of distributions: weighted orbital integrals", Duke Math. J. 99.2 (1999), 209-283.

[Art2] J. Arthur, "A note on L-packets", Pure Appl. Math. Quaterly 2.1 (2006), 199-217.

[Art3] J. Arthur, The endoscopic classification of representations: orthogonal and symplectic groups, Colloquium Publications volume 61, American Mathematical Society, 2013.

[Ati] M.F. Atiyah, K-theory, Mathematics Lecture Note Series, W.A. Benjamin, 1967.

[ABP] A.-M. Aubert, P.F. Baum, R.J. Plymen, "The Hecke algebra of a reductive p-adic group: a view from noncommutative geometry", pp. 1-34 in: Noncommutative geometry and number theory, Aspects of Mathematics E37, Vieweg Verlag, 2006.

[ABPS1] A.-M. Aubert, P.F. Baum, R.J. Plymen, M. Solleveld, "On the local Langlands correspondence for non-tempered representations", Münster Journal of Mathematics 7 (2014), 27-50.

[ABPS2] A.-M. Aubert, P.F. Baum, R.J. Plymen, M. Solleveld, "Geometric structure in smooth dual and local Langlands conjecture", Japanese Journal of Mathematics 9 (2014), 99-136.

[ABPS3] A.-M. Aubert, P.F. Baum, R.J. Plymen, M. Solleveld, "The local Langlands correspondence for inner forms of $\mathrm{SL}_{n} "$, arXiv:1305.2638, 2013.

[ABPS4] A.-M. Aubert, P.F. Baum, R.J. Plymen, M. Solleveld, "Hecke algebras for inner forms of p-adic special linear groups", J. Inst. Math. Jussieu (2015), 1-69.

[ABPS5] A.-M. Aubert, P.F. Baum, R.J. Plymen, M. Solleveld, "Geometric structure for the principal series of a split reductive $p$-adic group with connected centre", preprint, 2014, to appear in JNCG.

[ABPS6] A.-M. Aubert, P.F. Baum, R.J. Plymen, M. Solleveld, "The principal series of p-adic groups with disconnected centre", arXiv:1409.8110, 2014.

[ABPS7] A.-M. Aubert, P.F. Baum, R.J. Plymen, M. Solleveld, "The noncommutative geometry of inner forms of $p$-adic special linear groups", arXiv:1505.04361, 2015. 
[AHP] A.-M. Aubert, S. Hasan, R.J. Plymen, "Cycles in the chamber homology of GL(3)", KTheory 37 (2006), 341-377.

[AMS] A.-M. Aubert, A. Moussaoui, M. Solleveld, "Generalizations of the Springer correspondence and cuspidal Langlands parameters", arXiv:1511.05335, 2015

[Bad] A.I. Badulescu, "Correspondance de Jacquet-Langlands pour les corps locaux de caractéristique non nulle", Ann. Scient. Éc. Norm. Sup. 4e série 35 (2002), 695-747.

[BCH] P.F. Baum, A. Connes, N. Higson, "Classifying space for proper actions and K-theory of group C*-algebras" pp. 240-291 in: $C^{*}$-algebras: 1943-1993. A fifty year celebration, Contemp. Math. 167, American Mathematical Society, 1994.

[BHP1] P. Baum, N. Higson, R.J. Plymen, "Equivariant homology for SL(2) of a p-adic field", Contemp. Math. 148 (1993), 1-18.

[BHP2] P.F. Baum, N. Higson, R.J. Plymen, "Representation theory of $p$-adic groups: a view from operator algebras", Proc. Sympos. Pure. Math. 68 (2000), 111-149.

[BeDe] J. Bernstein, P. Deligne, "Le "centre" de Bernstein", pp. 1-32 in: Représentations des groupes réductifs sur un corps local, Travaux en cours, Hermann, 1984

[Bor1] A. Borel, "Admissible representations of a semi-simple group over a local field with vectors fixed under an Iwahori subgroup", Inv. Math. 35 (1976), 233-259.

[Bor2] A. Borel, "Automorphic L-functions", Proc. Symp. Pure Math 33.2 (1979), 27-61.

[BrPl] J. Brodzki, R.J. Plymen, "Geometry of the smooth dual of GL(n)", C.R. Acad. Sci. Paris 331 (2000), 213-218.

[Bro] K.S. Brown, Buildings, Springer Verlag, 1989.

[BrTi1] F. Bruhat, J. Tits, "Groupes réductifs sur un corps local: I. Données radicielles valuées", Publ. Math. Inst. Hautes Études Sci. 41 (1972), 5-251.

[BrTi2] F. Bruhat, J. Tits, "Groupes réductifs sur un corps local: II. Schémas en groupes. Existence d'une donnée radicielle valuée", Publ. Math. Inst. Hautes Études Sci. 60 (1984), 5-184.

[BuHe] C.J. Bushnell, G. Henniart, The local Langlands conjecture for GL(2), Grundlehren der mathematischen Wissenschaften 335, Springer Verlag, 2006.

[BuKu1] C.J. Bushnell, P.C. Kutzko, The admissible dual of $G L(N)$ via compact open subgroups, Annals of Mathematics Studies 129, Princeton University Press, 1993.

[BuKu2] C.J. Bushnell, P.C. Kutzko, "Smooth representations of reductive $p$-adic groups: structure theory via types", Proc. London Math. Soc. 77.3 (1998), 582-634.

[CuRe] C.W. Curtis, I. Reiner, Representation theory of finite groups and associative algebras, Pure and Applied Mathematics 11, John Wiley \& Sons, 1962

[Del] P. Deligne, "Les constantes des équations functionelles des fonctions L", Lecture Notes in Math. 349 (1973), 501-595.

[DKV] P. Deligne, D. Kazhdan, M.-F. Vigneras, "Représentations des algèbres centrales simples p-adiques", pp. 33-117 in: Représentations des groupes réductifs sur un corps local, Travaux en cours, Hermann, 1984.

[GoRo] D. Goldberg, A. Roche, "Hecke algebras and $S L_{n}$-types", Proc. London Math. Soc. 90.1 (2005), 87-131.

[GrRe] B.H. Gross, M. Reeder, "Arithmetic invariants of discrete Langlands parameters", Duke Math. J. 154.3 (2010), 431-508.

[HaTa] M. Harris, R. Taylor, The geometry and cohomology of some simple Shimura varieties, Annals of Math. Studies 151, Princeton University Press, 2001.

[Hai] T.J. Haines, "The stable Bernstein center and test functions for Shimura varieties", pp. 118186 in: Automorphic Forms and Galois Representations, London Mathematical Society Lecture Note Series 415, Cambrige University Press, 2014.

[H-C] Harish-Chandra, "Harmonic analysis on reductive p-adic groups", pp. 167-192 in: Harmonic analysis on homogeneous spaces, Proc. Sympos. Pure Math. 26, American Mathematical Society, 1973.

[Hei] V. Heiermann, "Opérateurs d'entrelacement et algèbres de Hecke avec paramètres d'un groupe réductif $p$-adique - le cas des groupes classiques", Selecta Math. 17.3 (2011), 713-756.

[Hen] G. Henniart, "Une preuve simple des conjectures de Langlands pour $G L(n)$ sur un corps p-adique", Inv. Math. 139 (2000), 439-455.

[HiNi] N. Higson, V. Nistor "Cyclic homology of totally disconnected groups acting on buildings", J. Funct. Anal. 141.2 (1996), 466-495. 
[HII] K. Hiraga, A. Ichino, T. Ikeda, "Formal degrees and adjoint $\gamma$-factors", J. Amer. Math. Soc. 21.1 (2008), 283-304 and correction J. Amer. Math. Soc. 21.4 (2008), 1211-1213.

[HiSa] K. Hiraga, H. Saito, "On L-packets for inner forms of $S L_{n}$ ", Mem. Amer. Math. Soc. 1013, Vol. 215 (2012).

[IwMa] N. Iwahori, H. Matsumoto, "On some Bruhat decomposition and the structure of the Hecke rings of the $p$-adic Chevalley groups", Inst. Hautes Études Sci. Publ. Math 25 (1965), 5-48.

[Jul] P. Julg, "K-théorie équivariante et produits croisés", C.R. Acad. Sci. Paris 292 (1981), 629632.

[Kal] T. Kaletha, "Epipelagic L-packets and rectifying characters", Inv. Math. (2015), DOI 10.1007/s00222-014-0566-4.

[KMSW] T. Kaletha, A. Minguez, S. W. Shin, P.-J. White, "Endoscopic classification of representations: inner forms of unitary groups", arXiv:1409.3731, 2014.

[KaLu] D. Kazhdan, G. Lusztig, "Proof of the Deligne-Langlands conjecture for Hecke algebras", Invent. Math. 87 (1987), 153-215.

[Kna] A. Knapp, "Introduction to the Langlands program", in: Representation theory and automorphic forms (Edinburgh, 1996), Proc. Sympos. Pure Math. 61 (1997), 245-302.

[Kot] R.E. Kottwitz, "Stable trace formula: cuspidal tempered terms", Duke Math. J. 51.3 (1984), 611-650.

[Laf1] V. Lafforgue, "K-théorie bivariante pour les algèbres de Banach et conjecture de BaumConnes", Invent. Math. 149.1 (2002), 1-95.

[Laf2] V. Lafforgue, "Chtoucas pour les groupes réductifs et paramétrisation de Langlands globale", arXiv:1209.5352, 2012.

[Laf3] V. Lafforgue, "Introduction to chtoucas for reductive groups and to the global Langlands parameterization", arXiv:1404.6416, 2014.

[Lan1] R.P. Langlands, "On the classification of irreducible representations of real algebraic groups", pp. 101-170 in: Representation theory and harmonic analysis on semisimple Lie groups, Math. Surveys Monogr. 31, American Mathematical Society, 1989.

[Lan2] R.P. Langlands, "Representations of abelian algebraic groups", pp. 231-250 in: Olga Taussky-Todd: in memoriam, Pacific J. Math., Special Issue, 1997.

[LRS] G. Laumon, M. Rapoport, U. Stuhler, "D-elliptic sheaves and the Langlands correspondence", Invent. Math. 113 (1993), 217-238.

[Lus1] G. Lusztig, "Some examples of square-integrable representations of semisimple $p$-adic groups", Trans. Amer. Math. Soc. 277.2 (1983), 623-653.

[Lus2] G. Lusztig, "Intersection cohomology complexes on a reductive group", Invent. Math. 75.2 (1984), 205-272.

[Lus3] G. Lusztig, "Cells in affine Weyl groups IV", J. Fac. Sci. Univ. Tokyo 36.2 (1989), 297-328.

[Lus4] G. Lusztig, "Affine Hecke algebras and their graded version", J. Amer. Math. Soc 2.3 (1989), 599-635.

[Lus5] G. Lusztig, "Classification of unipotent representations of simple p-adic groups", Int. Math. Res. Notices 11 (1995), 517-589.

[Lus6] G. Lusztig, "Classification of unipotent representations of simple p-adic groups II", Represent. Theory 6 (2002), 243-289.

[MiSt] M. Miyauchi, S. Stevens, "Semisimple types for $p$-adic classical groups", Math. Ann. 358 (2014), 257-288.

[Mok] C.P. Mok, "Endoscopic classification of representations of quasi-split unitary groups", Memoirs of the American Mathematical Society Vol. 235, Nr. 1108, 2015.

[Mor] L. Morris, "Tamely ramified intertwining algebras", Invent. Math. 114.1 (1993), 1-54.

[Mou] A. Moussaoui, "Centre de Bernstein stable et conjecture d'Aubert-Baum-PlymenSolleveld", PhD. thesis, Université Pierre et Marie Curie, 2015

[Opd] E.M. Opdam, "On the spectral decomposition of affine Hecke algebras", J. Inst. Math. Jussieu 3.4 (2004), 531-648.

[Phi] N.C. Phillips Equivariant K-theory and freeness of group actions on $C^{*}$-algebras, Lecture Notes in Mathematics 1274, Springer-Verlag, 1987.

[Ply] R. J. Plymen, "Reduced C*-algebra for reductive p-adic groups", J. Funct. Anal. 88.2 (1990), 251-266. 
[Ren] D. Renard, Représentations des groupes réductifs p-adiques, Cours spécialisés 17, Société Mathématique de France, 2010.

[Roc] A. Roche, "Types and Hecke algebras for principal series representations of split reductive p-adic groups", Ann. Sci. École Norm. Sup. 31.3 (1998), 361-413.

[Schn] P. Schneider, "Equivariant homology for totally disconnected groups", J. Algebra 203 (1998), 50-68.

[Scho] P. Scholze, "The local Langlands correspondence for $G L_{n}$ over $p$-adic fields", Invent. Math. 192 (2013), 663-715.

[Séc] V. Sécherre', "Représentations lisses de $G L_{m}(D)$ III: types simples", Ann. Scient. Éc. Norm. Sup. 38 (2005), 951-977.

[SéSt] V. Sécherre, S. Stevens, "Smooth representations of $G L(m, D)$ VI: semisimple types", Int. Math. Res. Notices 13 (2012), 2994-3039.

[SiZi] A.J. Silberger, E.-W. Zink, "Langlands Classification for L-Parameters", arXiv:1407.6494, 2014.

[Sol1] M. Solleveld, "Periodic cyclic homology of reductive $p$-adic groups", J. Noncommutative Geometry 3.4 (2009), 501-558.

[Sol2] M. Solleveld, "On the classification of irreducible representations of affine Hecke algebras with unequal parameters", Representation Theory 16 (2012), 1-87.

[Spr] T.A. Springer, Linear algebraic groups 2nd ed., Progress in Mathematics 9, Birkhäuser, 1998.

[Tit] J. Tits, "Reductive groups over local fields", pp. 29-69 in: Automorphic forms, representations and L-functions Part I, Proc. Sympos. Pure Math. 33, American Mathematical Society, 1979.

[Vig] M.-F. Vignéras, "On formal dimensions for reductive p-adic groups", pp. 225-266 in: Festschrift in honor of I.I. Piatetski-Shapiro on the occasion of his sixtieth birthday, Part I, Israel Math. Conf. Proc. 2, Weizmann, 1990.

[Vog] D. Vogan, "The local Langlands conjecture", pp. 305-379 in: Representation theory of groups and algebras, Contemp. Math. 145, American Mathematical Society, 1993.

[Voi] C. Voigt, "Chern character for totally disconnected groups", Math. Ann. 343.3 (2009), 507540.

[Wal] J.-L. Waldspurger, "La formule de Plancherel pour les groupes p-adiques (d'après HarishChandra)", J. Inst. Math. Jussieu 2.2 (2003), 235-333.

[Wei] A. Weil, Basic number theory 3rd ed., Grundlehren der mathematischen Wissenschaften 144, Springer-Verlag, 1974.

[Xu] B. Xu, "L-packets for quasi-split $\operatorname{GSp}(2 n)$ and $\mathrm{GO}(2 n)$ ", arXiv:1503.04897, 2015.

[Zel] A.V. Zelevinsky, "Induced representations of reductive p-adic groups II. On irreducible representations of GL(n)", Ann. Sci. École Norm. Sup. (4) 13.2 (1980), 165-210.

Institut de Mathématiques de Jussieu - Paris Rive Gauche, U.M.R. 7586 Du C.N.R.S., U.P.M.C., 4 Place Jussieu 75005 Paris, France

E-mail address: anne-marie.aubert@imj-prg.fr

Mathematics Department, Pennsylvania State University, University Park, PA 16802, USA

E-mail address: pxb6@psu.edu

School of Mathematics, Southampton University, Southampton SO17 1BJ, England and School of Mathematics, Manchester University, Manchester M13 9PL, England

E-mail address: r.j.plymen@soton.ac.uk plymen@manchester.ac.uk

IMAPP, Radboud Universiteit NiJmegen, HeyendaAlseweg 135, 6525AJ Nijmegen, the NETHERLANDS

E-mail address: m.solleveld@science.ru.nl 